\title{
Institutional semantics for many-valued logics
}

\author{
Răzvan Diaconescu \\ Simion Stoilow Institute of Mathematics of the Romanian Academy
}

\begin{abstract}
We develop many-valued logic, including a generic abstract model theory, over a fully abstract syntax. We show that important many-valued logic model theories, such as traditional first-order many-valued logic and fuzzy multi-algebras, may be conservatively embedded into our abstract framework. Our development is technically based upon the so-called theory of institutions of Goguen and Burstall and may serve as a template for defining at hand many-valued logic model theories over various concrete syntaxes or, from another perspective, to combine many-valued logic with other logical systems. We also show that our generic many-valued logic abstract model theory enjoys a couple of important institutional model theory properties that support the development of deep model theory methods.
\end{abstract}

Key words: institutions, many-valued logic

\section{Introduction}

Many-valued logic (abbreviated $m v l$; also known as 'multiple-valued' or 'multi-valued' logic) has a long tradition $[18,26,30]$ and needs no presentation. Our paper builds on the idea that the essence of mvl is actually independent of the concrete syntactic context in which it is usually presented, that in fact it is independent of any syntactic context. We realize this idea by developing mvl over a fully abstract syntax by employing the conceptual machinery of the so-called institution theory of Goguen and Burstall [19]. This is a categorical abstract model theory that arose about three decades ago within specification theory as a response to the explosion in the population of logics in use there, its original aim being to develop as much computing science as possible in a general uniform way independently of particular logical systems. This has now been achieved to an extent even greater than orginally thought, as institution theory became the most fundamental mathematical theory underlying algebraic specification theory (in its wider meaning) [33], also being increasingly used in other area of computer science. Moreover, institution theory constitutes a major trend in the so-called 'universal logic' (in the sense envisaged by Jean-Yves Béziau [2, 3]) which is considered by many a true renaissance of mathematical logic. A lot of model theory has gradually been developed at the level abstract institutions (see [10]).

The technical side of our abstract mvl development may be briefly described as follows. Given an abstract category of signatures and an abstract sentence functor (that gives the sets of sentences corresponding to the signatures) we build both a syntax and a model theory as well as a satisfaction relation between them.

- The given syntax is considered as atomic syntax and the full syntax is built by iterative applications of connectives $(\wedge, \vee, \Rightarrow$, etc.) and quantifiers. Quantifiers are also treated rather abstractly by the concept of quantification space of [11]; this covers concrete quantification situations much beyond first order.

Email address: Razvan.Diaconescueimar.ro (Răzvan Diaconescu)

Preprint submitted to Fuzzy Sets and Systems

November 26, 2012 
- The model theory is defined generically by a comma category construction plus an interpretation of a corresponding atomic syntax into a fixed space of truth values considered here as a fixed complete residuated lattice $L$.

- For each signature, the corresponding satisfaction relation between its models and its sentences is defined by recursion on the structure of the sentences in the usual Tarski style.

We show that this construction yields an institution by proving the so-called Satisfaction Condition of institution theory.

The generic abstract mvl thus developed (denoted $\mathcal{I}(L)$ ) covers various important concrete mvl systems. We show that the semantics of traditional first-order mvl (such as in [22]; here denoted $M V L$ ) may be conservatively embedded into $\mathcal{I}(L)$, which means that in this case the semantic consequence relation provided (generically) by $\mathcal{I}(L)$ coincides with that of $M V L$. This applies also to various restrictions or extensions of $M V L$, i.e. propositional fragment, second order extension, etc. A similar result is shown for a rather natural fuzzy extension of the multi-algebras framework [23, 24, 35, 36].

Moreover our generic abstract mvl development may be used as a semantic oriented framework for defining in an uniform way new concrete many-valued logical systems over various different logic syntaxes. This also means an uniform semantic oriented method to export mvl to other logical contexts. This has a special relevance within the current trend of logic combination, which has both theoretical and practical significance. The latter is related to the influential Goguen thesis [20] that the combinations of the computing paradigms is based upon the combinations of their underlying logical systems.

The abstract model theory emerging from our work, although abstract is still customized to the specificities of mvl. It also has the potential to support the development of deep model theoretic methods and results since we show that it enjoys rather naturally a couple of properties that pervade a lot of institutionindependent model theory developments [10]. These are model amalgamation and (the method of) diagrams. The in-depth development of a model theory at this level of abstraction brings not only the benefit of clarity but also that of generality. Any concrete many-valued model theory derived from our abstract framework would automatically enjoy the properties developed at the general level.

Finally, familiarity from the side of the reader with the abstract categorical view is quite essential for a proper understanding of this work.

\section{The structure of the paper}

1. The first technical section is devoted to the introduction of the institution theory elements that are necessary for our paper.

2. In the next section we define our abstract mvl framework $\mathcal{I}(L)$ and prove that it is an institution.

3. Then we show that traditional first order mvl and fuzzy multi-algebras are conservatively embedded into $\mathcal{I}(L)$.

4. The final technical section is devoted to showing that $\mathcal{I}(L)$ has model amalgamation and diagrams.

\section{Preliminaries}

The structure of the section is as follows:

1. We first introduce some basic category theory notations and terminology that are needed by our work.

2. Then we recall briefly a few of basic institution theory concepts that will be used in the paper. 
3. We illustrate the definition of institution by the presentation in some detail of classical first order logic with equality and of traditional first order many-valued logic, both in many sorted format and both captured as institutions. Another aim of this part is to fix some notations and terminology that concern the level of concrete logics and that will be used in the paper.

\subsection{Categories}

Our work is technically based upon institution theory which in turn is heavily based upon category theory. We assume the reader is familiar with basic notions and standard notations from category theory; e.g. [25]. Here we recall very briefly some of them. $|\mathbb{C}|$ denotes the class of objects of a category $\mathbb{C}$, composition is denoted by ";" and in diagrammatic order. The category of sets (as objects) and functions (as arrows) is denoted by Set, and CAT is the category of all categories. ${ }^{1}$ The opposite of a category $\mathbb{C}$ (obtained by reversing the arrows of $\mathbb{C}$ ) is denoted $\mathbb{C}^{\text {op }}$. For any object $A$ in a category $|\mathbb{C}|$, the comma category $A / \mathbb{C}$ has arrows $f: A \rightarrow B$ as objects and $h \in \mathbb{C}\left(B, B^{\prime}\right)$ with $f ; h=f^{\prime}$ as arrows $f \rightarrow f^{\prime}$.

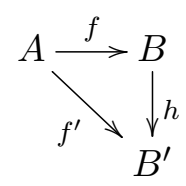

\subsection{Institutions}

Institutions have been defined by Goguen and Burstall in [5], the seminal paper [19] being printed after a delay of many years. Below we recall the concept of institution which formalises the intuitive notion of logical system, including syntax, semantics, and the satisfaction between them.

Definition 2.1 (Institution). An institution $\mathcal{I}=\left(\operatorname{Sign}^{\mathcal{I}}, \operatorname{Sen}^{\mathcal{I}}, \operatorname{Mod}^{\mathcal{I}},\left(\models \models_{\Sigma}^{\mathcal{I}}\right)_{\Sigma \in \mid \operatorname{Sign}^{\mathcal{I}}}\right)$ consists of

- a category $\operatorname{Sign}^{\mathcal{I}}$ whose objects are called signatures,

- a functor $\operatorname{Sen}^{\mathcal{I}}: \operatorname{Sign}^{\mathcal{I}} \rightarrow$ Set giving for each signature a set whose elements are called sentences over that signature,

- a functor $\mathrm{Mod}^{\mathcal{I}}:\left(\operatorname{Sign}^{\mathcal{I}}\right)^{o p} \rightarrow \mathrm{CAT}$, giving for each signature $\Sigma$ a category whose objects are called $\Sigma$-models, and whose arrows are called $\Sigma$-(model) homomorphisms, and

- a relation $\models \frac{\mathcal{I}}{\Sigma} \subseteq\left|\operatorname{Mod}^{\mathcal{I}}(\Sigma)\right| \times \operatorname{Sen}^{\mathcal{I}}(\Sigma)$ for each $\Sigma \in\left|\operatorname{Sign}^{\mathcal{I}}\right|$, called the satisfaction relation,

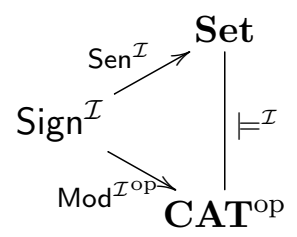

such that for each morphism $\varphi: \Sigma \rightarrow \Sigma^{\prime} \in \operatorname{Sign}^{\mathcal{I}}$, the Satisfaction Condition

$$
M^{\prime} \models=\frac{\mathcal{I}}{\Sigma^{\prime}} \operatorname{Sen}^{\mathcal{I}}(\varphi)(\rho) \text { if and only if } \operatorname{Mod}^{\mathcal{I}}(\varphi)\left(M^{\prime}\right) \models \stackrel{\mathcal{I}}{\Sigma} \rho
$$

\footnotetext{
${ }^{1}$ Strictly speaking, this is only a 'quasi-category' living in a higher set-theoretic universe.
} 
holds for each $M^{\prime} \in\left|\operatorname{Mod}^{\mathcal{I}}\left(\Sigma^{\prime}\right)\right|$ and $\rho \in \operatorname{Sen}^{\mathcal{I}}(\Sigma)$.

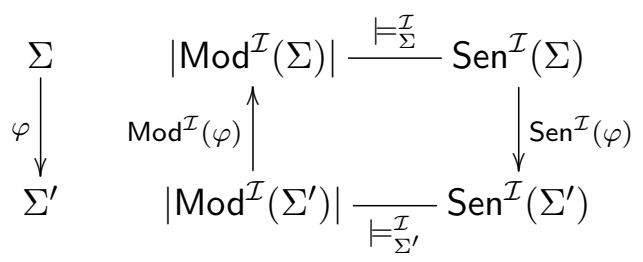

Notation 2.1. In any institution as above we use the following notations:

- for any $E \subseteq \operatorname{Sen}(\Sigma), E^{*}$ denotes $\left\{M \in|\operatorname{Mod}(\Sigma)| \mid M \models_{\Sigma} \rho\right.$ for each $\left.\rho \in E\right\}$.

- for any $E, E^{\prime} \subseteq \operatorname{Sen}(\Sigma), E \models E^{\prime}$ denotes $E^{*} \subseteq E^{\prime *}$.

- for any $E \subseteq \operatorname{Sen}(\Sigma), \operatorname{Mod}(\Sigma, E)$ is the full subcategory of $\operatorname{Mod}(\Sigma)$ whose objects are in $E^{*}$.

The following results constitute a simplified variant, sufficient for the purpose of this work, of well know results from the theory of institution comorphisms (e.g. [10,21]).

Proposition 2.1. For any institutions $\mathcal{I}$ and $\mathcal{I}^{\prime}$ such that $\operatorname{Sign}^{\mathcal{I}}=\operatorname{Sign}^{\mathcal{I}^{\prime}}$, for any signature $\Sigma$ and any $\alpha_{\Sigma}: \operatorname{Sen}^{\mathcal{I}}(\Sigma) \rightarrow \operatorname{Sen}^{\mathcal{I}^{\prime}}(\Sigma)$ if there exists $\beta_{\Sigma}:\left|\operatorname{Mod}^{\mathcal{I}^{\prime}}(\Sigma)\right| \rightarrow\left|\operatorname{Mod}^{\mathcal{I}}(\Sigma)\right|$ such that for each $\mathcal{I} \Sigma$-sentence $\rho$ and each $\mathcal{I}^{\prime} \Sigma$-model $M^{\prime}$

$\beta_{\Sigma}\left(M^{\prime}\right) \models=\frac{\mathcal{I}}{\Sigma} \rho$ if and only if $M^{\prime} \models \sum_{\Sigma}^{\mathcal{I}^{\prime}} \alpha_{\Sigma}(\rho)$,

then for any sets $E$ and $\Gamma$ of $\mathcal{I} \Sigma$-sentences

$E \models \frac{\mathcal{I}}{\Sigma} \Gamma$ implies $\alpha_{\Sigma}(E) \models_{\Sigma}^{\mathcal{I}^{\prime}} \alpha_{\Sigma}(\Gamma)$.

Moreover if there exists $\beta_{\Sigma}^{\prime}:\left|\operatorname{Mod}^{\mathcal{I}}(\Sigma)\right| \rightarrow\left|\operatorname{Mod}^{\mathcal{I}^{\prime}}(\Sigma)\right|$ such that

1. for each $\mathcal{I} \Sigma$-model $M, \beta_{\Sigma}\left(\beta_{\Sigma}^{\prime}(M)\right)=M$, or

2. for each $\mathcal{I} \Sigma$-model $M$ and each $\mathcal{I} \Sigma$-sentence $\rho$

$$
\beta_{\Sigma}^{\prime}(M) \models \sum_{\Sigma}^{\mathcal{I}^{\prime}} \alpha_{\Sigma}(\rho) \text { if and only if } M \models \models_{\Sigma}^{\mathcal{I}} \rho,
$$

then

$E \models{ }_{\Sigma}^{\mathcal{I}} \Gamma$ if and only if $\alpha_{\Sigma}(E) \models_{\Sigma}^{\mathcal{I}^{\prime}} \alpha_{\Sigma}(\Gamma)$.

\subsection{Examples of institutions}

Myriads of logical systems from computing or from mathematical logic have been captured as institutions; in fact it is thesis underlying institution theory that anything that deserves the name 'logic' should be captured by Dfn. 2.1. Below we recall rather briefly a couple of them that will also be used in examples in our paper. The first example may be the most representative institution in mathematical logic and computer science, while the other one may be the most representative within the context of mvl.

Example 2.1 (FOL). Let $F O L$ be the institution of first order logic with equality in its many sorted form. Its signatures (i.e. the objects of $\operatorname{Sign}^{F O L}$ ) are triples $(S, F, P)$ consisting of

- a set of sort symbols $S$, 
- a family $F=\left\{F_{w \rightarrow s} \mid w \in S^{*}, s \in S\right\}$ of sets of function symbols indexed by arities ( $w$, for the arguments; here $S^{*}$ denotes the words or strings formed with the symbols from $S$ ) and sorts ( $s$, for the results), and

- a family $P=\left\{P_{w} \mid w \in S^{*}\right\}$ of sets of relation (predicate) symbols indexed by arities.

Signature morphisms (i.e. the arrows of $\operatorname{Sign}^{F O L}$ ) map the three components in a compatible way. This means that a signature morphism $\varphi:(S, F, P) \rightarrow\left(S^{\prime}, F^{\prime}, P^{\prime}\right)$ consists of

- a function $\varphi^{\text {st }}: S \rightarrow S^{\prime}$,

- a family of functions $\varphi^{\mathrm{op}}=\left\{\varphi_{w \rightarrow s}^{\mathrm{op}}: F_{w \rightarrow s} \rightarrow F_{\varphi^{\mathrm{st}}(w) \rightarrow \varphi^{\mathrm{st}}(s)}^{\prime} \mid w \in S^{*}, s \in S\right\}$, and

- a family of functions $\varphi^{\mathrm{rl}}=\left\{\varphi_{w \rightarrow s}^{\mathrm{rl}}: P_{w} \rightarrow P_{\varphi^{\mathrm{st}}(w)}^{\prime} \mid w \in S^{*}\right\}$.

Models $M$ for a signature $(S, F, P)$ (i.e. the objects of $\left.\operatorname{Mod}^{F O L}(S, F, P)\right)$ are first order structures interpreting each sort symbol $s$ as a set $M_{s}$, each function symbol $\sigma \in F_{w \rightarrow s}$ as a function $M_{\sigma: w \rightarrow s}: M_{w} \rightarrow$ $M_{s}$ (often denoted just $M_{\sigma}$ when there is no danger of ambiguity) from the product of the interpretations of the argument sorts to the interpretation of the result sort, and each relation symbol $\pi$ as a subset $M_{\pi}$ of the product of the interpretations of the argument sorts. A model homomorphism $h: M \rightarrow M^{\prime}$ (i.e. an arrow in $\left.\operatorname{Mod}^{F O L}(S, F, P)\right)$ is an indexed family of functions $\left(h_{s}: M_{s} \rightarrow M_{s}^{\prime}\right)_{s \in S}$ such that

- $h$ is an $(S, F)$-algebra homomorphism $M \rightarrow M^{\prime}$, i.e., $h_{s}\left(M_{\sigma}(m)\right)=M_{\sigma}^{\prime}\left(h_{w}(m)\right)$ for each $\sigma \in$ $F_{w \rightarrow s}$ and each $m \in M_{w}$, and

- $h_{w}(m) \in M_{\pi}^{\prime}$ if $m \in M_{\pi}$ (i.e. $h_{w}\left(M_{\pi}\right) \subseteq M_{\pi}^{\prime}$ ) for each relation $\pi \in P_{w}$ and each $m \in M_{w}$.

where $h_{w}: M_{w} \rightarrow M_{w}^{\prime}$ is the canonical component-wise extension of $h$, i.e.

$$
h_{w}\left(m_{1}, \ldots, m_{n}\right)=\left(h_{s_{1}}\left(m_{1}\right), \ldots, h_{s_{n}}\left(m_{n}\right)\right) \text { for } w=s_{1} \ldots s_{n} \text { and } m_{i} \in M_{s_{i}} \text { for } 1 \leq i \leq n .
$$

For each signature morphism $\varphi$, the $\varphi$-reduct $\operatorname{Mod}(\varphi)\left(M^{\prime}\right)$ of a model $M^{\prime}$ is defined by $\operatorname{Mod}(\varphi)\left(M^{\prime}\right)_{x}=$ $M_{\varphi(x)}^{\prime}$ for each sort, function, or relation symbol $x$ from the domain signature of $\varphi$. Then $M^{\prime}$ is called a $\varphi$-expansion of $M$. When $\varphi$ is an inclusion of signatures $(S, F, P) \subseteq\left(S^{\prime}, F^{\prime}, P^{\prime}\right)$ then we may denote $\operatorname{Mod}(\varphi)\left(M^{\prime}\right)$ by $M^{\prime} \uparrow_{(S, F, P)}$.

Sentences are the usual first order sentences built from equational and relational atoms by iterative application of Boolean connectives and quantifiers, i.e.

- $t=t^{\prime}$ is an $(S, F, P)$-sentence when $t$ and $t^{\prime}$ are $(S, F)$-terms of the same sort; $\sigma\left(t_{1}, \ldots, t_{n}\right)$ is an $(S, F)$-term of sort $s$ when $\sigma \in F_{s_{1} \ldots s_{n} \rightarrow s}$ and $t_{1}, \ldots, t_{n}$ are $(S, F)$-terms of sorts $s_{1}, \ldots, s_{n}$ respectively. The set of the $(S, F)$-terms of sort $s$ is denoted $\left(T_{(S, F)}\right)_{s}$.

- $\pi\left(t_{1}, \ldots, t_{n}\right)$ is an $(S, F, P)$-sentence when $\pi \in P_{s_{1} \ldots s_{n}}$ and $t_{1}, \ldots, t_{n}$ are $(S, F)$-terms of sorts $s_{1}, \ldots, s_{n}$ respectively.

- $\rho_{1} \star \rho_{2}$ is an $(S, F, P)$-sentence if $\rho_{1}$ and $\rho_{2}$ are $(S, F, P)$-sentences and when $\star \in\{\wedge, \vee, \Rightarrow\}$.

- $\neg \rho$ is $(S, F, P)$-sentence if $\rho$ is $(S, F, P)$-sentence.

- A finite block $X$ of variables for $(S, F, P)$ is a set of triples of the form $(x, s,(S, F, P))$ where $x$ is the name of the variable and $s \in S$ is the sort of the variable and that two different variables in $X$ have different names. We often abbreviate variables $(x, s,(S, F, P))$ by their name $x$ or by their name and sort qualification like $(x: s)$. Then we let $(S, F+X, P)$ be the extension of $(S, F, P)$ 
such that $(F+X)_{w \rightarrow s}=F_{w \rightarrow s}$ when $w$ is non-empty and $(F+X)_{\rightarrow s}=F_{\rightarrow s} \cup\{(x, s,(S, F, P)) \mid$ $(x, s,(S, F, P)) \in X\}$.

Then $(\forall X) \rho$ and $(\exists X) \rho$ are $(S, F, P)$-sentences if $\rho$ is $(S, F+X, P)$-sentence where $X$ is a finite block of variables for $(S, F, P)$.

Sentence translations along signature morphisms just rename the sorts, function, and relation symbols according to the respective signature morphisms. They can be formally defined by recursion on the structure of the sentences. While the induction step is straightforward for the case of the Boolean connectives it needs a bit of attention for the case of the quantifiers. For any signature morphism $\varphi:(S, F, P) \rightarrow\left(S^{\prime}, F^{\prime}, P^{\prime}\right)$,

$$
\operatorname{Sen}^{F O L}(\varphi)((\forall X) \rho)=\left(\forall X^{\varphi}\right) \operatorname{Sen}^{F O L}\left(\varphi^{\prime}\right)(\rho)
$$

and we let $\varphi^{\prime}:(S, F+X, P) \rightarrow\left(S^{\prime}, F^{\prime}+X^{\varphi}, P^{\prime}\right)$ be the canonical extension of $\varphi$ that maps each variable $(x, s,(S, F, P))$ to $\left(x, \varphi(s),\left(S^{\prime}, F^{\prime}, P^{\prime}\right)\right)$. Note that because of the rather extensive qualification of the variables, Sen ${ }^{F O L}$ thus defined is functorial indeed and that there is no overloading of variables or clash between variables and the original constants of the signatures (which in certain situations would cause a failure of the Satisfaction Condition). All these in spite of the fact that we treat variables as constants. Also our treatment of the variables is 'localized' to corresponding signatures; this contrasts the common treatment of variables in logic which considers a fixed infinite set of variables from which the variables used in quantifications are picked at need. Our 'localized' approach match the practice of formal specification languages (e.g. $[1,6,14]$ etc.) in which the variables are declared and exist locally within the context of specification modules.

The satisfaction of sentences by models is the usual Tarskian satisfaction defined recursively on the structure of the sentences as follows:

- $M \models_{(S, F, P)} t=t^{\prime}$ when $M_{t}=M_{t^{\prime}}$, where $M_{t}$ denotes the interpretation of the $(S, F)$-term $t$ in $M$ defined recursively by

$$
M_{\sigma\left(t_{1}, \ldots, t_{n}\right)}=M_{\sigma}\left(M_{t_{1}}, \ldots, M_{t_{n}}\right) .
$$

- $M \models_{(S, F, P)} \pi\left(t_{1}, \ldots, t_{n}\right)$ when $\left(M t_{1}, \ldots, M_{t_{n}}\right) \in M_{\pi}$, for each relational atom $\pi\left(t_{1}, \ldots, t_{n}\right)$.

- $M \models_{(S, F, P)} \rho_{1} \wedge \rho_{2}$ when $M \models_{(S, F, P)} \rho_{1}$ and $M \models_{(S, F, P)} \rho_{2}$, and similarly for the other Boolean connectives $\vee, \Rightarrow, \neg$, etc.

- $M \models_{(S, F, P)}(\forall X) \rho$ when $M^{\prime} \models_{(S, F+X, P)} \rho$ for any $(S, F+X, P)$-expansion $M^{\prime}$ of $M$, and similarly for $\exists$.

The proof of the FOL Satisfaction Condition may be found in many places in the literature, e.g. [10].

Example 2.2 (Many-valued logic). This institution denoted $M V L$, of great tradition in non-classical logic $[18,26,30]$ and logical basis for 'fuzzy' developments, generalizes ordinary logic based upon the two Boolean truth values, true and false, to larger sets of truth values that are structured by the concept of residuated lattices [17, 22, 37].

Definition 2.2 (Residuated lattice). A residuated lattice $L$ is a bounded lattice (with $\leq$ denoting the underlying partial order that has infimum $\wedge$, supremum $\vee$, biggest $\top$ and lowest $\perp$ elements) and which comes equipped with an additional commutative and associative binary operation $\otimes$ which has $\top$ as identity and such that for all elements $x, y$ and $z$ 
$-(x \otimes y) \leq(x \otimes z)$ if $y \leq z$, and

- there exists an element $x \Rightarrow z$ such that $y \leq(x \Rightarrow z)$ if and only if $x \otimes y \leq z$.

The first condition of Dfn. 2.2 just means that $x \otimes-$ is a functor on the partial order $(L, \leq)$, and the second condition means that it has a left adjoint $x \Rightarrow-$. The ordinary two-valued situation can be recovered when $L$ is the two values Boolean algebra with $\otimes$ being the conjunction. Then $\Rightarrow$ is the ordinary Boolean implication. There is a myriad of interesting examples of residuated lattices used for many-valued logics for which $\otimes$ gets an interpretation rather different from the ordinary conjunction. One famous such example is the so-called Lukasiewicz arithmetic conjunction on the closed interval $[0,1]$ defined by $x \otimes y=1-$ $\min \{1,2-(x+y)\}$. In this example $x \Rightarrow y=\min \{1,1-x+y\}$.

Our presentation below of many-valued logic as institution is the same as in [13] and follows the ideas initially developed in [7]. Let us fix a residuated lattice $L$ that is also complete, i.e. it has infimum and supremum for any sets of elements. Our definition of the $M V L$ institution is implicitly parameterized by $L$.

$M V L$ signatures are just $F O L$ signatures $(S, F, P)$; likewise for the signature morphisms.

The $(S, F, P)$-sentences are pairs $(\rho, x)$ where $\rho$ is a quasi-sentence and $x$ is any element of $L$. The $(S, F, P)$-quasi-sentences are like the $F O L$ sentences, but instead of equational and relational atoms they are constructed only from relational atoms; moreover the usual set of connectives $\wedge, \vee, \Rightarrow$ is extended with connectives $\perp, \top \otimes$. The negation connector $\neg$ is missing as it may be defined in terms of $\Rightarrow$ and $\perp$. Like in $F O L$, in $M V L$ we also have universal $(\forall X)$ and existential $(\exists X)$ quantifications for finite sets $X$ of variables. Our formalization of the $M V L$-sentence as pairs between conventional sentences and truth values owes to the representation of the many-valued logic satisfaction relation, which is inherently a ternary relation, as a binary satisfaction relation as required by the concept of institution.

An $M V L(S, F, P)$-model $M$ is an $(S, F)$-algebra together with an interpretation of each relation symbol $\pi \in P_{w}$ as an L-relation, i.e. a function $M_{\pi}: M_{w} \rightarrow L$. A model homomorphism $h: M \rightarrow N$ is an $(S, F)$-algebra homomorphism such that $M_{\pi}(m) \leq N_{\pi}\left(h_{w}(m)\right)$ for each $\pi \in P_{w}$ and each $m \in M_{w}$.

For each $(S, F, P)$-model $M$ and each $(S, F, P)$-quasi-sentence $\rho$ we define a value $M \models \rho$ in $L$ as follows:

$$
\begin{aligned}
& \text { - }\left(M \models \pi\left(t_{1}, \ldots, t_{n}\right)\right)=M_{\pi}\left(M_{t_{1}}, \ldots, M_{t_{n}}\right) \text { for relational atoms, } \\
& \text { - }(M=\top)=\top \text { and }(M \models \perp)=\perp, \\
& \text { - }\left(M=\rho_{1} \star \rho_{2}\right)=\left(M \models \rho_{1}\right) \star\left(M \models \rho_{2}\right) \text { for } \star \in\{\wedge, \vee, \otimes, \Rightarrow\}, \\
& \text { - }(M \models(\forall X) \rho)=\bigwedge\left\{M^{\prime} \models \rho \mid M^{\prime} \uparrow_{(S, F, P)}=M\right\}, \text { and } \\
& \text { - }(M \models(\exists X) \rho)=\bigvee\left\{M^{\prime} \models \rho \mid M^{\prime} \uparrow_{(S, F, P)}=M\right\} .
\end{aligned}
$$

The translation of sentences and the model reducts along signature morphisms are defined like in FOL.

Then the $M V L$ satisfaction relation is defined by

$$
M \models \underset{(S, F, P)}{M V L}(\rho, x) \text { if and only if } x \leq(M \models \rho) .
$$

Note that the satisfaction relation $M \models \rho$ defined in [22] corresponds here to $M \models(\rho, \top)$. A proof of the MVL Satisfaction Condition may be found in [7]. The propositional many-valued logic institution is obtained as the 'sub-institution' of $M V L$ determined by restricting the signatures to those that have the set of sort symbols empty. This means the signatures are sets $P$ of zero arity relation symbols and that consequently the models are just valuations $P \rightarrow L$. The sentences are just terms over $P \cup\{\top, \perp\}$ formed with the binary operators $\wedge, \vee, \Rightarrow, \otimes$. 


\section{Abstract many-valued institutions}

In this section we develop the generic many-valued institution $\mathcal{I}(L)$ that may serve as an abstract framework for embedding concrete many valued institutions. The structure of the section is as follows:

1. We introduce some technical notions necessary for defining $\mathcal{I}(L)$.

2. We give the definition of $\mathcal{I}(L)$.

3. We prove the Satisfaction Condition for $\mathcal{I}(L)$.

\subsection{Technical preliminaries}

The following concept has been first introduced in [11].

Definition 3.1 (Quantification space). For any category Sign a subclass of arrows $\mathcal{D} \subseteq$ Sign is called a quantification space if, for any $\left(\chi: \Sigma \rightarrow \Sigma^{\prime}\right) \in \mathcal{D}$ and $\varphi: \Sigma \rightarrow \Sigma_{1}$, there is a designated pushout

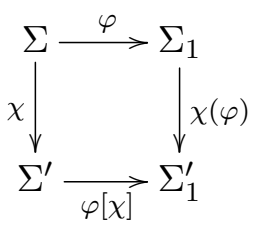

with $\chi(\varphi) \in \mathcal{D}$ and such that the 'horizontal' composition of such designated pushouts is again a designated pushout, i.e. for the pushouts in the following diagram

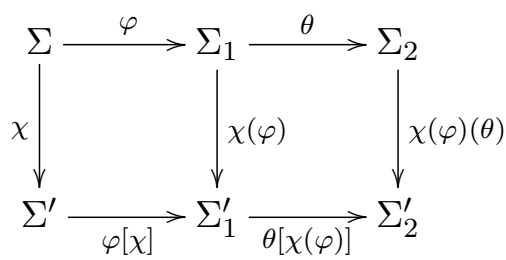

$\varphi[\chi] ; \theta[\chi(\varphi)]=(\varphi ; \theta)[\chi]$ and $\chi(\varphi)(\theta)=\chi(\varphi ; \theta)$, and such that $\chi\left(1_{\Sigma}\right)=\chi$ and $1_{\Sigma}[\chi]=1_{\Sigma^{\prime}}$

Example 3.1. Within the context of Ex. 2.1 above, the signature extensions $\chi:(S, F, P) \hookrightarrow(S, F+X, P)$, where $X$ is a finite block of variables for $(S, F, P)$ constitute a quantification space for $\operatorname{Sign}^{F O L}$ that is adequate for $\operatorname{Mod}^{F O L}$. Given signature morphism $\varphi:(S, F, P) \rightarrow\left(S_{1}, F_{1}, P_{1}\right)$, then

- $\chi(\varphi):\left(S_{1}, F_{1}, P_{1}\right) \hookrightarrow\left(S_{1}, F_{1}+X^{\varphi}, P_{1}\right)$ where $X^{\varphi}$ as defined in Ex. 2.1, and

- $\varphi[\chi]$ is the canonical extension of $\varphi$ that maps each $(x, s,(S, F, P))$ to $\left(x, \varphi^{\text {st }}(s),\left(S_{1}, F_{1}, P_{1}\right)\right)$ (it corresponds to $\varphi^{\prime}$ of Ex. 2.1).

It is easy to note that these define pushout squares fulfilling the properties of Dfn. 3.1.

Other quantification spaces for Sign ${ }^{F O L}$ may be obtained as follows:

1. In the example above we consider infinite blocks of variables instead of finite ones.

2. We consider blocks of second order variables of the form $(x,(w, s),(S, F, P))$ (function variables) or of the form $(x, w,(S, F, P))$ (relation variables) where $w \in S^{*}$ and $s \in S$. Then to any block $X$ of second order variables it corresponds a signature extension $\chi:(S, F, P) \rightarrow\left(S, F+X^{\mathrm{op}}, P+X^{\mathrm{rl}}\right)$ where $X$ is split as $X^{\mathrm{op}} \cup X^{\mathrm{rl}}$ with $X^{\mathrm{op}}$ being the function variables and $X^{\mathrm{rl}}$ the relation variables, and where $F+X^{\mathrm{op}}$ and $P+X^{\mathrm{rl}}$ extend in the obvious way the definition of $F+X$ from Ex. 2.1. 
Note that these definitions may also apply to $M V L$.

We borrow the following terminology from [31]:

Definition 3.2. A logic syntax is a pair (Sign, Sen) such that

1. Sign is a category whose objects are called 'signatures' and whose arrows are called 'signature morphisms', and

2. a functor Sen: Sign $\rightarrow$ Set called 'sentence functor'; the objects of $\operatorname{Sen}(\Sigma)$ are called ' $\Sigma$-sentences'.

\subsection{The definition of $\mathcal{I}(L)$}

In the following, given a residuated lattice $L$ and a tuple (Sign, FSen, CSen, $\left.\mathrm{CSen}_{0}, \mathcal{D}\right)$ such that

1. (Sign, FSen) is a logic syntax (called the fuzzy atomic syntax),

2. (Sign, CSen) is a logic syntax (called the crisp atomic syntax),

3. the fuzzy and the crisp atomic syntaxes are disjoint, i.e. for each signature $\Sigma$,

$$
\operatorname{FSen}(\Sigma) \cap \operatorname{CSen}(\Sigma)=\emptyset
$$

4. CSen $0 \subseteq$ CSen is a sub-functor (called the crisp truth functor), and

5. $\mathcal{D}$ is a quantification space for Sign

we develop a generic institution $\mathcal{I}(L)$. The role of CSen is to provide an explicit syntax for crisp satisfaction (see Dfn. 3.5 below) which widens the range of applications for $\mathcal{I}(L)$. This crisp component of $\mathcal{I}(L)$ is crucial for covering specific mvl systems with an explicit crisp component (like in Remark 4.2 below) or even without (like in Dfn. 4.1) below.

Definition $3.3(\mathcal{I}(L)$ models). For any signature $\Sigma$, a $\Sigma$-model is a pair $(\mu, m)$ that consists of

- a signature morphism $\mu: \Sigma \rightarrow \Sigma_{\mu}$, and

- a function $m: \operatorname{FSen}\left(\Sigma_{\mu}\right) \rightarrow L$.

A $\Sigma$-homomorphism $h:(\mu, m) \rightarrow(\nu, n)$ between two $\Sigma$-models is a signature morphism $h: \Sigma_{\mu} \rightarrow \Sigma_{\nu}$ such that $\mu ; h=\nu$ and $m \leq \mathrm{FSen}(h) ; n$.
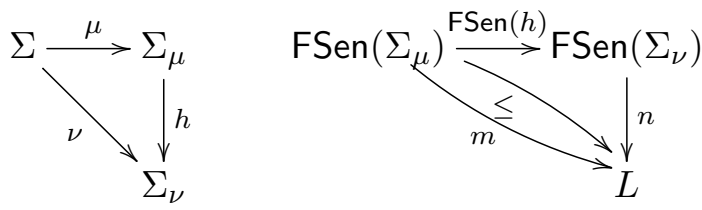

The category of the $\Sigma$-homomorphisms (composition inherited from Sign) is denoted $\operatorname{Mod}^{\mathcal{I}(L)}(\Sigma)$.

For any signature morphism $\varphi: \Sigma \rightarrow \Sigma^{\prime}$ the $\varphi$-reduct $\operatorname{Mod}^{\mathcal{I}(L)}(\varphi)\left(\mu^{\prime}, m^{\prime}\right)$ of a $\Sigma^{\prime}$-model $\left(\mu^{\prime}, m^{\prime}\right)$ is $\left(\varphi ; \mu^{\prime}, m^{\prime}\right)$. The $\varphi$-reduct $\operatorname{Mod}^{\mathcal{I}(L)}(\varphi)\left(h^{\prime}\right)$ of a $\Sigma^{\prime}$-homomorphism $h^{\prime}:\left(\mu^{\prime}, m^{\prime}\right) \rightarrow\left(\nu^{\prime}, n^{\prime}\right)$ is just $h^{\prime}:\left(\varphi ; \mu^{\prime}, m^{\prime}\right) \rightarrow\left(\varphi ; \nu^{\prime}, n^{\prime}\right)$.

Fact 3.1. For any signature morphism $\varphi: \Sigma \rightarrow \Sigma^{\prime}, \operatorname{Mod}^{\mathcal{I}(L)}(\varphi): \operatorname{Mod}^{\mathcal{I}(L)}\left(\Sigma^{\prime}\right) \rightarrow \operatorname{Mod}^{\mathcal{I}(L)}(\Sigma)$ is functor. Moreover, $\operatorname{Mod}^{\mathcal{I}(L)}$ is a functor $\operatorname{Sign} \rightarrow$ CAT $^{\text {op }}$. 
In the concrete situations the component $\mu$ from the definition of $\mathcal{I}(L)$ models is usually a signature extension with a set of constants representing the carrier (or underlying) set of the respective model. The component $m$ interprets the atomic syntax corresponding to the extended signature into the space $L$ of the truth values. Note that the crisp atomic syntax does not play any role in the semantics of $\mathcal{I}(L)$. These considerations may be understood fully when studying the examples provided in Sect. 4 below, in which the fuzzy syntax captures relations while the crisp syntax captures equations.

Definition $3.4(\mathcal{I}(L)$ sentences). Let Sen* be the least mapping Sign $\rightarrow$ Set such that for each signature $\Sigma$

- $\operatorname{FSen}(\Sigma) \cup \operatorname{CSen}(\Sigma) \subseteq \operatorname{Sen}^{*}(\Sigma)$

- $T, \perp \in \operatorname{Sen}^{*}(\Sigma)$,

- $\rho_{1} \star \rho_{2} \in \operatorname{Sen}^{*}(\Sigma)$ for $\star \in\{\wedge, \vee, \Rightarrow, \otimes\}$ and for all $\rho_{1}, \rho_{2} \in \operatorname{Sen}^{*}(\Sigma)$, and

- $(\forall \chi) \rho,(\exists \chi) \rho \in \operatorname{Sen}^{*}(\Sigma)$ for all $\rho \in \operatorname{Sen}^{*}\left(\Sigma^{\prime}\right)$ and each $\left(\chi: \Sigma \rightarrow \Sigma^{\prime}\right) \in \mathcal{D}$,

and for each signature morphism $\varphi: \Sigma \rightarrow \Sigma_{1}$

- $\operatorname{Sen}^{*}(\varphi)(\rho)=\mathrm{FSen}(\varphi)(\rho)$ for each $\rho \in \mathrm{FSen}(\Sigma)$,

- $\operatorname{Sen}^{*}(\varphi)(\top)=\top$, $\operatorname{Sen}^{*}(\varphi)(\perp)=\perp$,

- $\operatorname{Sen}^{*}(\varphi)(\rho)=\operatorname{CSen}(\varphi)(\rho)$ for each $\rho \in \operatorname{CSen}(\Sigma)$,

- $\operatorname{Sen}^{*}(\varphi)\left(\rho_{1} \star \rho_{2}\right)=\operatorname{Sen}^{*}(\varphi)\left(\rho_{1}\right) \star \operatorname{Sen}^{*}(\varphi)\left(\rho_{2}\right)$ for $\star \in\{\wedge, \vee, \Rightarrow, \otimes\}$ and for all $\rho_{1}, \rho_{2} \in \operatorname{Sen}^{*}(\Sigma)$, and

- $\operatorname{Sen}^{*}(\varphi)((\forall \chi) \rho)=(\forall \chi(\varphi)) \operatorname{Sen}^{*}(\varphi[\chi])(\rho)$ for each $\rho \in \operatorname{Sen}^{*}\left(\Sigma^{\prime}\right)$ and similarly for $(\exists \chi) \rho$.

Let $\operatorname{Sen}^{\mathcal{I}(L)}=$ Sen $^{*} \times L$ be the mapping Sign $\rightarrow$ Set defined by

- $\operatorname{Sen}^{\mathcal{I}(L)}(\Sigma)=\operatorname{Sen}^{*}(\Sigma) \times L$ for each signature $\Sigma$, and

- $\operatorname{Sen}^{\mathcal{I}(L)}(\varphi)(\rho, x)=\left(\operatorname{Sen}^{*}(\varphi)(\rho), x\right)$ for each signature morphism $\varphi: \Sigma \rightarrow \Sigma_{1}$, each $\Sigma$-quasisentence $\rho$ and each $x \in L$.

Note that $\perp$ and $T$ are used with two different meanings: on the one hand as truth values (i.e. elements of $L$ ), and on the other hand as sentences. We think this overloading of notation may actually help the reader because of the relationship between the two meanings; the truth value of the sentences $\perp$ or $T$ is always exactly the corresponding element of $L$.

Proposition 3.1. $\operatorname{Sen}^{\mathcal{I}(L)}$ is functor.

Proof. This is an immediate consequence of the functoriality of Sen* which is obtained as follows by recursion on the structure of the sentence $\rho$.

When $\rho \in \mathrm{FSen}(\Sigma)$ :

$$
\begin{array}{rlrl}
\operatorname{Sen}^{*}(\varphi ; \theta)(\rho) & =\mathrm{FSen}(\varphi ; \theta)(\rho) & & \text { (by Dfn. 3.4) } \\
& =\mathrm{FSen}(\theta)(\mathrm{FSen}(\varphi)(\rho)) & \text { (functoriality of FSen }) \\
& =\operatorname{Sen}^{*}(\theta)\left(\operatorname{Sen}^{*}(\varphi)(\rho)\right) & & \text { (Dfn. 3.4). }
\end{array}
$$


The case when $\rho \in \operatorname{CSen}(\Sigma)$ is similar to the previous case.

The case when $\rho$ is $\perp$ or $T$ is trivial.

When $\rho=\rho_{1} \star \rho_{2}$ with $\star \in\{\wedge, \vee, \Rightarrow, \otimes\}$ :

$$
\begin{aligned}
\operatorname{Sen}^{*}(\varphi ; \theta)\left(\rho_{1} \star \rho_{2}\right) & =\operatorname{Sen}(\varphi ; \theta)\left(\rho_{1}\right) \star \operatorname{Sen}^{*}(\varphi ; \theta)\left(\rho_{2}\right) & & \text { (by Dfn. 3.4) } \\
& =\operatorname{Sen}^{*}(\theta)\left(\operatorname{Sen}^{*}(\varphi)\left(\rho_{1}\right)\right) \star \operatorname{Sen}^{*}(\theta)\left(\operatorname{Sen}^{*}(\varphi)\left(\rho_{2}\right)\right) & & \text { (recursion hypothesis) } \\
& =\operatorname{Sen}^{*}(\theta)\left(\operatorname{Sen}^{*}(\varphi)\left(\rho_{1}\right) \star \operatorname{Sen}^{*}(\varphi)\left(\rho_{2}\right)\right) & & \text { (by Dfn. 3.4) } \\
& =\operatorname{Sen}^{*}(\theta)\left(\operatorname{Sen}^{*}(\varphi)\left(\rho_{1} \star \rho_{2}\right)\right) & & \text { (by Dfn. 3.4). }
\end{aligned}
$$

When $\rho$ is $(\forall \chi) \rho^{\prime}$ with $\left(\chi: \Sigma \rightarrow \Sigma^{\prime}\right) \in \mathcal{D}$ and $\rho^{\prime} \in \operatorname{Sen}^{*}\left(\Sigma^{\prime}\right)$ :

$$
\begin{aligned}
\operatorname{Sen}^{*}(\varphi ; \theta)\left((\forall \chi) \rho^{\prime}\right) & =(\forall \chi(\varphi ; \theta)) \operatorname{Sen}^{*}((\varphi ; \theta)[\chi])\left(\rho^{\prime}\right) & & \text { (by Dfn. 3.4) } \\
& =(\forall \chi(\varphi)(\theta)) \operatorname{Sen}^{*}(\varphi[\chi] ; \theta[\chi(\varphi)])\left(\rho^{\prime}\right) & & \text { (by Dfn. 3.1) } \\
& =(\forall \chi(\varphi)(\theta)) \operatorname{Sen}^{*}(\theta[\chi(\varphi)])\left(\operatorname{Sen}^{*}(\varphi[\chi])\left(\rho^{\prime}\right)\right) & & \text { (by recursion hypothesis) } \\
& =\operatorname{Sen}^{*}(\theta)\left((\forall \chi(\varphi)) \operatorname{Sen}^{*}(\varphi[\chi])\left(\rho^{\prime}\right)\right) & & \text { (by Dfn. 3.4) } \\
& =\operatorname{Sen}^{*}(\theta)\left(\operatorname{Sen}^{*}(\varphi)\left((\forall \chi) \rho^{\prime}\right)\right) & & \text { (by Dfn. 3.4). }
\end{aligned}
$$

The case when $\rho$ is $(\exists \chi) \rho^{\prime}$ is similar to the previous case.

The $\mathcal{I}(L)$ satisfaction relation between models and sentences follows the usual recursive Tarski style:

Definition $3.5\left(\mathcal{I}(L)\right.$ satisfaction). For each signature $\Sigma$ we define a 'satisfaction degree' function $\left(\_-\right.$ -) : $\left|\operatorname{Mod}^{\mathcal{I}(L)}(\Sigma)\right| \times \operatorname{Sen}^{*}(\Sigma) \rightarrow L$ by

- for each $\rho \in \mathrm{FSen}(\Sigma),((\mu, m) \models \rho)=m(\operatorname{FSen}(\mu)(\rho))$,

- for each $\rho \in \operatorname{CSen}(\Sigma),((\mu, m) \models \rho)=\left\{\begin{array}{cl}\top & \text { when } \operatorname{CSen}(\mu)(\rho) \in \operatorname{CSen}_{0}\left(\Sigma_{\mu}\right) \text {, } \\ \perp & \text { otherwise. }\end{array}\right.$

- $((\mu, m) \models \top)=\top$ and $((\mu, m) \models \perp)=\perp$,

- $\left((\mu, m) \models \rho_{1} \star \rho_{2}\right)=\left((\mu, m) \models \rho_{1}\right) \star\left((\mu, m) \models \rho_{2}\right)$ for $\star \in\{\wedge, \vee, \Rightarrow, \otimes\}$,

- $((\mu, m) \models(\forall \chi) \rho)=\bigwedge\left\{\left(\mu^{\prime}, m\right) \models \rho \mid \mu^{\prime}: \Sigma^{\prime} \rightarrow \Sigma_{\mu}, \mu=\chi ; \mu^{\prime}\right\}$, and

- $((\mu, m) \models(\exists \chi) \rho)=\bigvee\left\{\left(\mu^{\prime}, m\right) \models \rho \mid \mu^{\prime}: \Sigma^{\prime} \rightarrow \Sigma_{\mu}, \mu=\chi ; \mu^{\prime}\right\}$.

Then for any $\Sigma$-model $(\mu, m)$ and any sentence $\rho \in \operatorname{Sen}^{\mathcal{I}(L)}(\Sigma)$,

$$
(\mu, m) \models_{\Sigma}^{\mathcal{I}(L)}(\rho, k) \text { if and only if } k \leq((\mu, m) \models \rho) .
$$

3.3. The Satisfaction Condition of $\mathcal{I}(L)$

Proposition 3.2. For any signature morphism $\varphi: \Sigma \rightarrow \Sigma_{1}$, any $\Sigma_{1}$-model $(\mu, m)$, and any $\rho \in \operatorname{Sen}^{*}(\Sigma)$

$$
((\varphi ; \mu, m) \models \rho)=\left((\mu, m) \models \operatorname{Sen}^{*}(\varphi)(\rho)\right) .
$$

Proof. We prove the conclusion of the proposition by recursion on the structure of $\rho$.

When $\rho \in \operatorname{FSen}(\Sigma)$ :

$$
\begin{aligned}
(\varphi ; \mu, m) \models \rho & =m(\mathrm{FSen}(\varphi ; \mu)(\rho)) & & \left(\text { definition of }(\varphi ; \mu, m) \models{ }_{-}\right) \\
& =m(\mathrm{FSen}(\mu)(\mathrm{FSen}(\varphi)(\rho))) & & \text { (functoriality of FSen) } \\
& =m\left(\mathrm{FSen}(\mu)\left(\operatorname{Sen}^{*}(\varphi)(\rho)\right)\right) & & \text { (definition of } \left.\operatorname{Sen}^{*}(\varphi)\right) \\
& =(\mu, m) \models \operatorname{Sen}^{*}(\varphi)(\rho) & & \left(\text { definition of }(\mu, m) \models{ }_{-}\right) .
\end{aligned}
$$


When $\rho \in \operatorname{CSen}(\Sigma)$ :

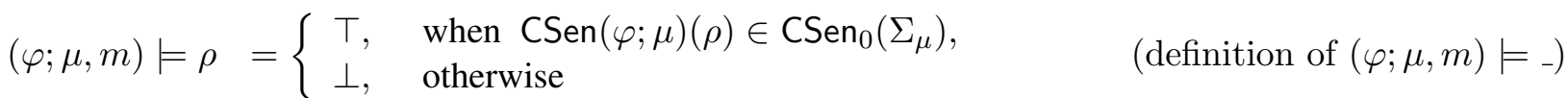

$$
\begin{aligned}
& = \begin{cases}\top, & \text { when } \operatorname{CSen}(\mu)(\operatorname{CSen}(\varphi)(\rho)) \in \operatorname{CSen}_{0}\left(\Sigma_{\mu}\right), \quad \text { (functoriality of CSen) } \\
\perp, & \text { otherwise }\end{cases} \\
& =(\mu, m)=\operatorname{CSen}(\varphi)(\rho) \\
& =(\mu, m)=\operatorname{Sen}^{*}(\varphi)(\rho) \\
& \text { (definition of }(\mu, m) \models-\text { ) } \\
& \text { (definition of } \operatorname{Sen}^{*}(\varphi) \text { ). }
\end{aligned}
$$

The case when $\rho \in\{\top, \perp\}$ is trivial.

When $\rho=\rho_{1} \star \rho_{2}$ with $\star \in\{\wedge, \vee, \Rightarrow, \otimes\}$ :

$$
\begin{aligned}
(\varphi ; \mu, m) \models \rho_{1} \star \rho_{2} & =\left((\varphi ; \mu, m) \models \rho_{1}\right) \star\left((\varphi ; \mu, m) \models \rho_{2}\right) & & \text { (definition of } \left.(\varphi ; \mu, m) \models_{-}\right) \\
& =\left((\mu, m) \models \operatorname{Sen}^{*}(\varphi)\left(\rho_{1}\right) \star \star\left((\mu, m) \models \operatorname{Sen}^{*}(\varphi)\left(\rho_{2}\right)\right)\right. & & \text { (recursion hypothesis) } \\
& =(\mu, m) \models \operatorname{Sen}^{*}(\varphi)\left(\rho_{1}\right) \star \operatorname{Sen}^{*}(\varphi)\left(\rho_{2}\right) & & \text { (definition of } \left.(\mu, m) \models-\models_{-}\right) \\
& =(\mu, m) \models \operatorname{Sen}^{*}(\varphi)\left(\rho_{1} \star \rho_{2}\right) & & \text { (definition of Sen*). }
\end{aligned}
$$

When $\rho$ is $(\forall \chi) \rho^{\prime}$ with $\left(\chi: \Sigma \rightarrow \Sigma^{\prime}\right) \in \mathcal{D}$ and $\rho^{\prime} \in \operatorname{Sen}^{*}\left(\Sigma^{\prime}\right)$ :

$$
\left((\varphi ; \mu, m) \models(\forall \chi) \rho^{\prime}\right)=\bigwedge\left\{\left(\mu^{\prime}, m\right) \models \rho^{\prime} \mid \mu^{\prime}: \Sigma^{\prime} \rightarrow\left(\Sigma_{1}\right)_{\mu}, \varphi ; \mu=\chi ; \mu^{\prime}\right\}
$$

(definition of $(\varphi ; \mu, m) \models$ _ and of reduct of $\mathcal{I}(L)$ models).

and

$$
\begin{aligned}
& \left((\mu, m) \models \operatorname{Sen}^{*}(\varphi)\left((\forall \chi) \rho^{\prime}\right)\right)=(\mu, m)\left((\forall \chi(\varphi)) \operatorname{Sen}^{*}(\varphi[\chi])\left(\rho^{\prime}\right)\right) \\
& \text { (definition of Sen*) } \\
& =\bigwedge\left\{\left(\mu_{1}, m\right) \models \operatorname{Sen}^{*}(\varphi[\chi])\left(\rho^{\prime}\right) \mid \mu_{1}: \Sigma_{1}^{\prime} \rightarrow\left(\Sigma_{1}\right)_{\mu}, \mu=\chi(\varphi) ; \mu_{1}\right\} \\
& \text { (definition of }(\mu, m) \models_{-} \text {) } \\
& =\bigwedge\left\{\left(\varphi[\chi] ; \mu_{1}, m\right) \models \rho^{\prime} \mid \mu_{1}: \Sigma_{1}^{\prime} \rightarrow\left(\Sigma_{1}\right)_{\mu}, \mu=\chi(\varphi) ; \mu_{1}\right\} \\
& \text { (recursion hypothesis). }
\end{aligned}
$$

One the one hand, for any $\mu^{\prime}: \Sigma^{\prime} \rightarrow\left(\Sigma_{1}\right)_{\mu}$ such that $\varphi ; \mu=\chi ; \mu^{\prime}$ by the pushout property of the square $(\varphi, \chi, \varphi[\chi], \chi(\varphi))$ below there exists $\mu_{1}: \Sigma_{1}^{\prime} \rightarrow\left(\Sigma_{1}\right)_{\mu}$ such that $\mu=\chi(\varphi) ; \mu_{1}$ and $\mu^{\prime}=\varphi[\chi] ; \mu_{1}$.

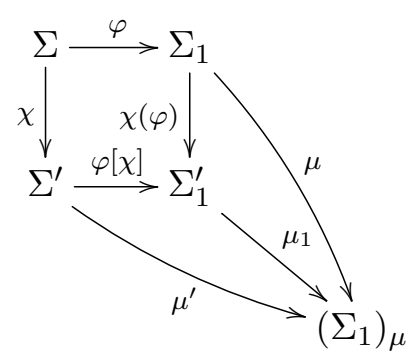

This implies that

$$
\left((\mu, m) \models \operatorname{Sen}^{*}(\varphi)\left((\forall \chi) \rho^{\prime}\right)\right) \leq\left((\varphi ; \mu, m) \models(\forall \chi) \rho^{\prime}\right) .
$$

On the other hand, for any $\mu_{1}: \Sigma_{1}^{\prime} \rightarrow\left(\Sigma_{1}\right)_{\mu}$ such that $\mu=\chi[\varphi] ; \mu_{1}$ we define $\mu^{\prime}=\varphi[\chi] ; \mu_{1}$. By the commutativity of the square $(\varphi, \chi, \varphi[\chi], \chi(\varphi))$ it follows that $\varphi ; \mu=\chi ; \mu^{\prime}$, hence

$$
\left((\varphi ; \mu, m) \models(\forall \chi) \rho^{\prime}\right) \leq\left((\mu, m) \models \operatorname{Sen}^{*}(\varphi)\left((\forall \chi) \rho^{\prime}\right)\right) .
$$


The conclusion of this case follows from (2) and (3). The case when $\rho$ is $(\exists \chi) \rho^{\prime}$ gets a similar treatment to the universal quantification case.

Corollary 3.1 $\left(\mathcal{I}(L)\right.$ satisfaction condition). (Sign, $\left.\operatorname{Sen}^{\mathcal{I}(L)}, \operatorname{Mod}^{\mathcal{I}(L)}, \models^{\mathcal{I}(L)}\right)$ is an institution.

Remark 3.1 (Relationship to [16]). The result of Prop. 3.2 shows that $\left(\operatorname{Sign}, \operatorname{Sen}^{*}, \operatorname{Mod}^{\mathcal{I}(L)},\left(\left|=_{\Sigma}:\right| \operatorname{Mod}^{\mathcal{I}(L)}(\Sigma) \mid \times\right.\right.$ $\left.\left.\operatorname{Sen}^{*}(\Sigma) \rightarrow L\right)_{\Sigma \in|\operatorname{Sign}|}\right)$ is a 'generalized institution' in sense of [16]. The partially ordered monad involved in this case would be the trivial identity monad over Set. This may be easily extended to an example based upon the power-set monad partially ordered by the subset inclusion relation just by extending $\mid=$ to sets of sentences by $(M \models \Gamma)=\bigwedge_{\rho \in \Gamma}(M \models \rho)$. The work [16] represents another abstract institution theoretic approach to mvl but which goes in a different direction than ours. The generalized institutions of [16] are fully abstract and represent a generalization of the concept of institution that essentially considers a many-valued satisfaction relation instead of a two-valued one as in the traditional definition of institutions [19]. However this is not the case here where we build a generic class of institutions on top of abstract atomic syntaxes. This retains the possibility of an in-depth development of generic model theory methods and results specific to mvl, which may not be possible at a higher level of abstraction in which most of the semantic specificities of mvl evaporate. Moreover we also take the step of capturing our generic mvl construction as a traditional two-valued institution in order to directly benefit from the rather rich institution theoretic developments in the literature, both in computer science and model theory. In fact this is a completely canonical step which may be undertaken for any generalized institution in the sense of [16] as follows.

Proposition 3.3. Given a complete lattice L, any generalized institution in the sense of [16], (Sign, Mod, Sen, $\Phi, \preccurlyeq, \eta, \mu, \models$ ), determines an institution (Sign, Mod, Sen ${ }^{\prime}, \models^{\prime}$ ) defined by

$$
\begin{aligned}
& -\operatorname{Sen}^{\prime}(\Sigma)=\operatorname{Sen}(\Sigma) \times L, \text { and } \\
& -M \models_{\Sigma}^{\prime}(\rho, x) \text { if and only if } x \leq\left(M \models_{\Sigma} \eta_{\operatorname{Sen}(\Sigma)}(\rho)\right) .
\end{aligned}
$$

Proof. We have just to show the Satisfaction Condition for $=^{\prime}$. Let $\varphi: \Sigma \rightarrow \Sigma^{\prime}$ be any signature morphism, let $M^{\prime}$ be any $\Sigma^{\prime}$-model and let $(\rho, x)$ be any $\Sigma$-sentence. Then

$$
\begin{aligned}
& M^{\prime} \models{ }^{\prime} \operatorname{Sen}^{\prime}(\varphi)(\rho, x) \text { iff } \quad M^{\prime} \models^{\prime}(\operatorname{Sen}(\varphi)(\rho), x) \quad \text { (definition of } \operatorname{Sen}^{\prime} \text { ) } \\
& \text { iff } \quad x \leq\left(M^{\prime} \models \eta_{\operatorname{Sen}\left(\Sigma^{\prime}\right)}(\operatorname{Sen}(\varphi)(\rho))\right) \quad \text { (definition of } \models^{\prime} \text { ) } \\
& \text { iff } \quad x \leq\left(M^{\prime} \models \Phi(\operatorname{Sen}(\varphi))\left(\eta_{\operatorname{Sen}(\Sigma)}(\rho)\right)\right) \quad \text { (naturality of } \eta \text { ) } \\
& \text { iff } x \leq\left(\operatorname{Mod}(\varphi)\left(M^{\prime}\right) \mid=\eta_{\operatorname{Sen}(\Sigma)}(\rho)\right) \\
& \text { (by the satisfaction condition in the generalized institution) } \\
& \text { iff } \operatorname{Mod}(\varphi)\left(M^{\prime}\right) \models^{\prime}(\rho, x) \quad \text { (definition of } \models^{\prime} \text { ). }
\end{aligned}
$$

Remark 3.2. In our construction of $\mathcal{I}(L)$ the residuated lattice $L$ is a fixed parameter. However one may easily extend this to have $L$ flexible by indexing by $L$ and then by considering a flattening construction as in [8]. In short this means that instead of considering a fixed $L$ and signatures $\Sigma$ we rather consider signatures of the form $(L, \Sigma)$. 


\section{Embedding concrete many-valued institutions into $\mathcal{I}(L)$}

In this section we show that two important concrete mvl systems, namely traditional first order mvl $(M V L)$ and a fuzzy extension of multi-algebras (of $[23,24,35,36])$, may be embedded conservatively into our abstract mvl framework $\mathcal{I}(L)$. These are embeddings both at the syntactic and at the semantic level. A consequence of such embeddings is that corresponding instances of the generic abstract model theory provided by $\mathcal{I}(L)$ may be used as a replacement for the respective original model theories. For $M V L$ we also discuss some of its variations, such as the propositional fragment and extensions with crisp equality and with second order quantifications.

The examples display slightly different situations: while in the $M V L$ case the embedding is bijective at the level of the syntax, in the fuzzy multi-algebras case it is bijective at the level of the semantics.

\subsection{MVL revisited}

In this example we show that, assuming a fixed residuated lattice $L$, the $M V L$ syntax together with its semantic consequence relation arise as the syntax and the semantic consequence relation of a corresponding instance of the generic institution $\mathcal{I}(L)$. For this we set the parameters of $\mathcal{I}(L)$ as follows:

- Sign $=\operatorname{Sign}^{M V L}\left(=\operatorname{Sign}^{F O L}\right)($ see Ex. 2.2),

$-\mathcal{D}$ consists of the signature extensions with finite blocks of first order variables as given by Ex. 3.1, and

- for each signature $(S, F, P)$, FSen $(S, F, P)$ consists of the set of all relational $(S, F, P)$-atoms, i.e.

$$
\operatorname{FSen}(S, F, P)=\left\{\pi\left(t_{1}, \ldots, t_{n}\right) \mid \pi \in P_{s_{1} \ldots s_{n}}, t_{1} \in\left(T_{(S, F)}\right)_{s_{1}}, \ldots,\left(T_{(S, F)}\right)_{s_{n}}\right\}
$$

and

- CSen is empty.

Fact 4.1. For each signature $(S, F, P)$ there is a canonical bijection $\alpha_{(S, F, P)}$ between the set of $(S, F, P)$ quasi-sentences and $\operatorname{Sen}^{*}(S, F, P)$ defined by

$$
\begin{aligned}
& -\alpha_{(S, F, P)}\left(\pi\left(t_{1}, \ldots, t_{n}\right)\right)=\pi\left(t_{1}, \ldots, t_{n}\right), \\
- & \alpha_{(S, F, P)}(\top)=\top, \alpha_{(S, F, P)}(\perp)=\perp, \\
- & \alpha_{(S, F, P)}\left(\rho_{1} \star \rho_{2}\right)=\alpha_{(S, F, P)}\left(\rho_{1}\right) \star \alpha_{(S, F, P)}\left(\rho_{2}\right) \text { for } \star \in\{\wedge, \vee, \Rightarrow, \otimes\}, \text { and } \\
- & \alpha_{(S, F, P)}((Q X) \rho)=(Q \chi) \alpha_{(S, F+X, P)}(\rho) \text { for any } Q \in\{\forall, \exists\} \text { and where } \chi \text { denotes the signature } \\
& \text { extension }(S, F, P) \rightarrow(S, F+X, P) .
\end{aligned}
$$

This bijection extends trivially to a bijection $\operatorname{Sen}^{M V L}(S, F, P) \cong \operatorname{Sen}^{\mathcal{I}(L)}(S, F, P)$; we denote the latter also by $\alpha_{(S, F, P)}$.

Proposition 4.1. For each signature $(S, F, P)$ there is a mapping $\beta_{(S, F, P)}:\left|\operatorname{Mod}^{\mathcal{I}(L)}(S, F, P)\right| \rightarrow\left|\operatorname{Mod}^{M V L}(S, F, P)\right|$ such that for each $M V L(S, F, P)$-sentence $\rho$ and each $\mathcal{I}(L)(S, F, P)$-model $(\mu, m)$

$$
\left(\beta_{(S, F, P)}(\mu, m) \models \rho\right)=\left((\mu, m) \models \alpha_{(S, F, P)}(\rho)\right) .
$$

Proof. Let us denote $\beta_{(S, F, P)}(\mu, m)$ by $M$; let $\mu:(S, F, P) \rightarrow\left(S^{\prime}, F^{\prime}, P^{\prime}\right)$. The definition of $M$ is as follows: 
- For each sort $s \in S, M_{s}=\left(T_{\left(S^{\prime}, F^{\prime}\right)}\right)_{\mu(s)}$.

- For each $\sigma \in F_{w \rightarrow s}, M_{\sigma}\left(t_{1}^{\prime}, \ldots, t_{n}^{\prime}\right)=\mu(\sigma)\left(t_{1}^{\prime}, \ldots, t_{n}^{\prime}\right)$.

- For each $\pi \in P_{w}, M_{\pi}\left(t_{1}^{\prime}, \ldots, t_{n}^{\prime}\right)=m\left(\mu(\pi)\left(t_{1}^{\prime}, \ldots, t_{n}^{\prime}\right)\right)$.

We prove relation (4) by recursion on the structure of $\rho$.

When $\rho$ is $\pi\left(t_{1}, \ldots, t_{n}\right)$ :

$$
\begin{aligned}
(\mu, m) \models \alpha_{(S, F, P)}\left(\pi\left(t_{1}, \ldots, t_{n}\right)\right) & =(\mu, m) \models \pi\left(t_{1}, \ldots, t_{n}\right) & & \left(\text { definition of } \alpha_{(S, F, P)}\right) \\
& =m\left(\mathrm{FSen}(\mu)\left(\pi\left(t_{1}, \ldots, t_{n}\right)\right)\right) & & \text { (definition of }(\mu, m) \models \\
& =m\left(\mu(\pi)\left(\mu\left(t_{1}\right), \ldots, \mu\left(t_{n}\right)\right)\right) & & \text { (definition of FSen }(\mu)) \\
& =M_{\pi}\left(\mu\left(t_{1}\right), \ldots, \mu\left(t_{n}\right)\right) & & \text { (definition of } \left.M_{\pi}\right) \\
& =M_{\pi}\left(M_{t_{1}}, \ldots, M_{t_{n}}\right) & & \text { (definition of term evaluation in } M \text { ) } \\
& =M \models \pi\left(t_{1}, \ldots, t_{n}\right) & & \text { (definition of } \left.M=_{-}\right) .
\end{aligned}
$$

When $\rho \in\{\top, \perp\}$ it is trivial.

When $\rho=\rho_{1} \star \rho_{2}$ with $\star \in\{\wedge, \vee, \Rightarrow, \otimes\}$ :

$$
\begin{array}{rlrl}
(\mu, m) \models \alpha_{(S, F, P)}\left(\rho_{1} \star \rho_{2}\right) & =(\mu, m) \models \alpha_{(S, F, P)}\left(\rho_{1}\right) \star \alpha_{(S, F, P)}\left(\rho_{2}\right) & & \text { (definition of } \left.\alpha_{(S, F, P)}\right) \\
& =\left((\mu, m) \models \alpha_{(S, F, P)}\left(\rho_{1}\right)\right) \star\left((\mu, m) \models \alpha_{(S, F, P)}\left(\rho_{2}\right)\right) & & \text { (definition of }(\mu, m) \models \\
& & \\
& =\left(M \models \rho_{1}\right) \star\left(M \models \rho_{2}\right) & & \text { (recursion hypothesis ) } \\
& =M \models \rho_{1} \star \rho_{2} & & \text { (definition of } \left.M \models \models_{-}\right) .
\end{array}
$$

When $\rho$ is $(\forall X) \rho^{\prime}$ :

Fact 4.2. $\beta_{(S, F+X, P)}$ gives a canonical bijection between the sets $\left\{\left(\mu^{\prime}, m\right) \mid \mu^{\prime}:(S, F+X, P) \rightarrow\right.$ $\left.\left(S^{\prime}, F^{\prime}, P^{\prime}\right), \mu=\chi ; \mu^{\prime}\right\}$ and $\left\{M^{\prime} \mid M^{\prime}\right.$ is $(S, F+X, P)$-expansion of $\left.M\right\}$ that is defined by the relation

$$
\begin{aligned}
M_{x}^{\prime}=\mu^{\prime}(x), \text { for all } x \text { in } X . & \\
(\mu, m) \models \alpha_{(S, F, P)}\left((\forall X) \rho^{\prime}\right)= & \left.(\mu, m) \models(\forall \chi) \alpha_{(S, F+X, P)}\left(\rho^{\prime}\right) \quad \text { (definition of } \alpha\right) \\
= & \bigwedge\left\{\left(\mu^{\prime}, m\right) \models \alpha_{(S, F+X, P)}\left(\rho^{\prime}\right) \mid \mu^{\prime}:(S, F+X, P) \rightarrow\left(S^{\prime}, F^{\prime}, P^{\prime}\right), \mu=\chi ; \mu^{\prime}\right\} \\
& (\text { definition of }(\mu, m) \models-) \\
= & \bigwedge\left\{M^{\prime} \models \rho^{\prime} \mid M^{\prime} \text { is }(S, F+X, P) \text {-expansion of } M\right\} \\
& (\text { Fact } 4.2 \text { and recursion hypothesis) } \\
= & \left.M \models(\forall X) \rho^{\prime} \text { (definition of } M \models-{ }_{-}\right) .
\end{aligned}
$$

When $\rho$ is $(\exists X) \rho^{\prime}$ we get a proof very similar to that of the universal quantification case above, hence we skip it here.

Proposition 4.2. For each signature $(S, F, P)$ there is a mapping $\beta_{(S, F, P)}^{\prime}:\left|\operatorname{Mod}^{M V L}(S, F, P)\right| \rightarrow\left|\operatorname{Mod}^{\mathcal{I}(L)}(S, F, P)\right|$ such that for each $M V L(S, F, P)$-sentence $\rho$ and each $M V L(S, F, P)$-model $M$

$$
\left(\beta_{(S, F, P)}^{\prime}(M) \models \alpha_{(S, F, P)}(\rho)\right)=(M \models \rho) .
$$

Proof. Let us denote $\beta_{(S, F, P)}^{\prime}(M)$ by $(\mu, m)$; its definition is as follows: 
- $\mu:(S, F, P) \rightarrow\left(S, F_{M}, P\right)$ such that

$-\mu^{\mathrm{st}}=1_{S}$ and $\mu_{w}^{\mathrm{rl}}=1_{P_{w}}$.

$-\left(F_{M}\right)_{w \rightarrow s}= \begin{cases}F_{w \rightarrow s} & \text { when } w \text { is non-empty, } \\ M_{s} & \text { when } w \text { is empty. }\end{cases}$

- for each $\sigma \in F_{w \rightarrow s}, \mu^{\mathrm{op}}(\sigma)= \begin{cases}\sigma & \text { when } w \text { is non-empty, } \\ M_{\sigma} & \text { when } w \text { is empty. }\end{cases}$

- $m\left(\pi\left(t_{1}^{\prime}, \ldots, t_{n}^{\prime}\right)\right)=M_{\pi}\left(M\left(t_{1}^{\prime}\right), \ldots, M\left(t_{n}^{\prime}\right)\right)$ where

$$
M\left(\sigma\left(t_{1}, \ldots, t_{k}\right)\right)= \begin{cases}\sigma & \text { when } k=0, \\ M_{\sigma}\left(M\left(t_{1}\right), \ldots, M\left(t_{k}\right)\right) & \text { when } k \neq 0 .\end{cases}
$$

Fact 4.3. For each $(S, F)$-term $t, M_{t}=M(\mu(t))$.

We prove relation (5) by recursion on the structure of $\rho$.

When $\rho$ is $\pi\left(t_{1}, \ldots, t_{n}\right)$ :

$$
\begin{aligned}
(\mu, m) \models \alpha_{(S, F, P)}\left(\pi\left(t_{1}, \ldots, t_{n}\right)\right) & =m\left(\mu(\pi)\left(\mu\left(t_{1}\right), \ldots, \mu\left(t_{n}\right)\right)\right) & & \text { (like in Prop. 4.1) } \\
& =m\left(\pi\left(\mu\left(t_{1}\right), \ldots, \mu\left(t_{n}\right)\right)\right) & & \text { ( } \mu^{\mathrm{rl}} \text { is identity) } \\
& =M_{\pi}\left(M\left(\mu\left(t_{1}\right)\right), \ldots, M\left(\mu\left(t_{n}\right)\right)\right) & & \text { (definition of } m) \\
& =M_{\pi}\left(M_{t_{1}}, \ldots, M_{t_{n}}\right) & & \text { (Fact 4.3) } \\
& =M=\pi\left(t_{1}, \ldots, t_{n}\right) & & \text { (definition of } \left.M \models_{-}\right) .
\end{aligned}
$$

When $\rho=\rho_{1} \star \rho_{2}$ with $\star \in\{\wedge, \vee, \Rightarrow, \otimes\}$ : like in Prop. 4.1.

When $\rho \in\{\top, \perp\}$ it is trivial.

When $\rho$ is $(\forall X) \rho^{\prime}$ : like in Prop. 4.1 using Fact 4.4 below instead of Fact 4.2.

Fact 4.4. $\beta_{(S, F+X, P)}^{\prime}$ gives a canonical bijection between the sets $\left\{\left(\mu^{\prime}, m\right) \mid \mu^{\prime}:(S, F+X, P) \rightarrow\right.$ $\left.\left(S, F_{M}, P\right), \mu=\chi ; \mu^{\prime}\right\}$ and $\left\{M^{\prime} \mid M^{\prime}\right.$ is $(S, F+X, P)$-expansion of $\left.M\right\}$ that is defined by the relation

$$
M_{x}^{\prime}=\mu^{\prime}(x) \text {, for all } x \text { in } X .
$$

The existential quantification case is treated similarly to the universal quantification.

By Prop. 4.1 and Prop. 4.2 we get the following straightforward instance of Prop. 2.1 which just shows that $M V L$ is conservatively embedded into $\mathcal{I}(L)$.

Corollary 4.1. For each signature $(S, F, P)$, for each set of $M V L(S, F, P)$-sentences $E$ and each $M V L$ $(S, F, P)$-sentence e

$$
E \models{ }_{(S, F, P)}^{M V L} e \text { if and only if } \alpha_{(S, F, P)}(E) \models_{(S, F, P)}^{\mathcal{I}(L)} \alpha_{(S, F, P)}(e) .
$$

Remark 4.1 (The second order extension). If we extended $M V L$ with second order quantifiers of the form $(\forall \pi) \rho$, where $\pi$ is a relation symbol, then we just have to modify the parameter $\mathcal{D}$ of $\mathcal{I}(L)$ above by letting $\mathcal{D}$ be wider: the class of the signature extensions with first order variables and with relation symbol variables. 
Remark 4.2 (Adding crisp equality). If we considered the extension of $M V L$ with crisp equality, which means adding equations $t=t^{\prime}$ with crisp satisfaction to the atomic sentences, then our encoding of $M V L$ into $\mathcal{I}(L)$ may be extended by upgrading the crisp atomic syntax from empty to

- $\operatorname{CSen}(S, F, P)=\left\{t=t^{\prime} \mid t, t^{\prime} \in\left(T_{(S, F)}\right)_{s}, s \in S\right\}$, and

- $\operatorname{CSen}_{0}(S, F, P)=\left\{t=t \mid t \in\left(T_{(S, F)}\right)_{s}, s \in S\right\}$.

and by extending the definition of $\alpha$ to crisp equations by $\alpha_{(S, F, P)}\left(t=t^{\prime}\right)=\left(t=t^{\prime}\right)$. It is rather easy to note that while (4) holds for the crisp equalities $t=t^{\prime}$, (5) does not. This means that only

$$
E \models{ }_{(S, F, P)}^{M V L} e \text { implies } \alpha_{(S, F, P)}(E) \models_{(S, F, P)}^{\mathcal{I}(L)} \alpha_{(S, F, P)}(e) .
$$

holds.

Remark 4.3 (Propositional many-valued logic). In the case of the propositional (fragment of) $M V L$ we may note that the corresponding variant of Cor. 4.1 holds as an instance of Prop. 2.1 without the need of the relation (5) because in this case we have that $\beta\left(\beta^{\prime}(M)\right)=M$ for each model $M$ of propositional $M V L$.

\subsection{Fuzzy multi-algebras}

In this example we show that the fuzzy extension of multi-algebras may be conservatively embedded into a corresponding instance of $\mathcal{I}(L)$; consequently the semantic consequence relation of the former arise as the semantic consequence of the latter. Moreover the semantic component of this embedding is a bijection.

Multi-algebras have been introduced as an algebraic specification framework for non-determinism [23, 35, 36] and later on developed as a general framework for algebraic specification [24]. Lamo's thesis [24] shows that multi-algebras subsume important algebraic formalisms used in formal specification such as partial algebra [4] and membership algebra [28]. The non-deterministic nature of the operations in multialgebras makes the logic of multi-algebras very suitable for a fuzzy generalization. In the following we first present the institution of fuzzy multi-algebras, with the classical multi-algebras being obtained for the two-valued lattice $L$. After this we build a conservative embedding of the institution of fuzzy multi-algebras into a corresponding instance of $\mathcal{I}(L)$.

Example 4.1 (The institution of fuzzy multi-algebras). We fix a residuated lattice $L$.

The signatures are triples $(S, F, C)$ where

- $S$ is a set (of sort symbols),

- $F$ is an indexed family $\left\{F_{w \rightarrow s} \mid w \in S^{*}, s \in S\right\}$ of sets (of operation symbols), and

$-C$ is an indexed family $\left\{C_{s} \mid s \in S\right\}$ (of deterministic constants).

Signature morphisms maps the three components in a compatible way like in FOL or $M V L$.

An $(S, F, C)$-model $M$ consists of

- for each sort $s \in S$, a set $M_{s}$,

- for each operation symbol $\sigma \in F_{w \rightarrow s}$, a function $M_{\sigma}: M_{w} \times M_{s} \rightarrow L$, and

- for each deterministic constant $c \in C_{s}$, an element $M_{c} \in M_{s}$. 
$(S, F, C)$-quasi-sentences are formed from atoms $t \prec t^{\prime}$ (with $t$ and $t^{\prime}$ being $(S, F+C)$-terms of the same sort) by iterative applications of connectives $(\wedge, \vee, \Rightarrow, \otimes)$ and quantifications with blocks of first order variables considered as (new) deterministic constants. ${ }^{2}$ The $(S, F, C)$-sentences are pairs formed by an $(S, F, C)$-quasi-sentence and an element of $L$.

For defining the satisfaction between models and sentences we first define for each $(S, F, C)$-model $M$ a term evaluation function $M\left[,_{-}\right]: T_{(S, F+C)} \times M \rightarrow L$ by the following recursive formula:

$M\left[\sigma\left(t_{1}, \ldots, t_{n}\right), a\right]=\left\{\begin{array}{l}\top, \text { when } \sigma \in C_{s}, M_{\sigma}=a, \\ \perp, \text { when } \sigma \in C_{s}, M_{\sigma} \neq a, \\ \bigvee\left\{M_{\sigma}\left(b_{1}, \ldots, b_{n}, a\right) \wedge M\left[t_{1}, b_{1}\right] \wedge \ldots \wedge M\left[t_{n}, b_{n}\right] \mid\left(b_{1}, \ldots, b_{n}\right) \in M_{w}\right\}, \text { when } \sigma \in F_{w \rightarrow s} .\end{array}\right.$

Then for each signature $\Sigma$ we define a function ${ }_{-} \models_{\Sigma}$ - that takes a $\Sigma$-model and a $\Sigma$-quasi-sentence and returns an element of $L$ as follows:

- $\left(M \mid=t \prec t^{\prime}\right)=\bigwedge\left\{M[t, a] \Rightarrow M\left[t^{\prime}, a\right] \mid a \in M\right\}$,

- $\left(M=\rho_{1} \star \rho_{2}\right)=\left(M \models \rho_{1}\right) \star\left(M \models \rho_{2}\right)$ for $\star \in\{\wedge, \vee, \otimes, \Rightarrow\}$,

- $(M \mid=(\forall X) \rho)=\bigwedge\left\{M^{\prime}|=\rho| M^{\prime} \uparrow_{(S, F, P)}=M\right\}$, and

- $(M \mid=(\exists X) \rho)=\bigvee\left\{M^{\prime}|\rho| M^{\prime} \uparrow_{(S, F, P)}=M\right\}$.

Like for $M V L$ (see Ex. 2.2) we use $M \models{ }_{-}$to define the satisfaction relation between models and sentences:

$M \models(\rho, k)$ if and only if $k \leq(M \models \rho)$.

The institution thus obtained is denoted FMALG. We omit here the proof of FMALG Satisfaction Condition which, given the encoding below, may be obtained from the corresponding instance of Prop. 3.2.

In order to embed FMALG into a corresponding instance of $\mathcal{I}(L)$ we set the parameters of $\mathcal{I}(L)$ as follows:

- Sign $=\operatorname{Sign}^{F M A L G}$,

- $\mathcal{D}$ consists of the signature extensions $(S, F, C) \subseteq(S, F, C+X)$ with finite blocks of first order variables as in Ex. 3.1, and

- for each signature $(S, F, C)$,

- FSen $(S, F, C)=\left\{a \prec \sigma\left(a_{1}, \ldots, a_{n}\right) \mid \sigma \in F_{s_{1} \ldots s_{n} \rightarrow s}, a_{1} \in C_{s_{1}}, \ldots, a_{n} \in C_{s_{n}}, a \in C_{s}\right\}$,

- $\operatorname{CSen}(S, F, C)=\left\{a=a^{\prime} \mid s \in S, a, a^{\prime} \in C_{s}\right\}$,

- $\operatorname{CSen}_{0}(S, F, C)=\left\{a=a \mid s \in S, a \in C_{s}\right\}$.

Definition 4.1. For each $F M A L G$ signature $(S, F, C)$ we define a translation $\alpha_{(S, F, C)}: \operatorname{Sen}^{F M A L G}(S, F, C) \rightarrow$ $\operatorname{Sen}^{\mathcal{I}(L)}(S, F, C)$ as follows:

\footnotetext{
${ }^{2}$ Works such as [24] employ also the deterministic equality $t \doteq t^{\prime}$ as atomic sentence, however we omit this here since it may be derived from the current syntax.
} 
- $\alpha_{(S, F, C)}\left(t \prec t^{\prime}\right)=(\forall \chi)\left(x \prec t \Rightarrow x \prec t^{\prime}\right)$ where for each $(S, F+C)$-term $t$ and $x$ deterministic constant, $x \prec t$ denotes the formula defined recursively by

$$
\left(x \prec \sigma\left(t_{1}, \ldots, t_{n}\right)\right)= \begin{cases}(\exists \Upsilon)\left(x \prec \sigma\left(y_{1}, \ldots, y_{n}\right) \wedge y_{1} \prec t_{1} \wedge \ldots \wedge y_{n} \prec t_{n}\right), & \text { when } \sigma \notin C_{s} \\ x=\sigma, & \text { when } \sigma \in C_{s} .\end{cases}
$$

and $\chi$ denotes the signature extension $(S, F, C) \subseteq(S, F, C+x)$ and $\Upsilon$ the signature extension $(S, F, C+x) \subseteq\left(S, F, C+x+\left\{y_{1}, \ldots, y_{n}\right\}\right)$,

$-\alpha_{(S, F, C)}\left(\rho_{1} \star \rho_{2}\right)=\alpha_{(S, F, C)}\left(\rho_{1}\right) \star \alpha_{(S, F, C)}\left(\rho_{2}\right)$,

- $\alpha_{(S, F, C)}((Q X) \rho)=(Q \chi) \alpha_{(S, F, C+X)}(\rho)$ for any $Q \in\{\forall, \exists\}$ and where $\chi$ denotes the signature extension $(S, F, C) \rightarrow(S, F, C+X)$, and

- $\alpha_{(S, F, C)}(\rho, k)=\left(\alpha_{(S, F, C)}(\rho), k\right)$.

Proposition 4.3. For each signature $(S, F, C)$ there exists a canonical bijection $\beta_{(S, F, C)}:\left|\operatorname{Mod}^{\mathcal{I}(L)}(S, F, C)\right| \rightarrow$ $\left|\operatorname{Mod}^{F M A L G}(S, F, C)\right|$ such that for each FMALG $(S, F, C)$-quasi-sentence $\rho$ and each $\mathcal{I}(L)(S, F, C)$ model $(\mu, m)$

$$
\left(\beta_{(S, F, C)}(\mu, m) \models \rho\right)=\left((\mu, m) \models \alpha_{(S, F, C)}(\rho)\right) .
$$

Proof. If $\mu:(S, F, C) \rightarrow\left(S^{\prime}, F^{\prime}, C^{\prime}\right)$ then $M=\beta_{(S, F, C)}(\mu, m)$ is defined as follows:

- for each $s \in S, M_{s}=C_{s}^{\prime}$,

- for each $\sigma \in F_{w \rightarrow s}, M_{\sigma}\left(a_{1}, \ldots, a_{n}, a\right)=m\left(a \prec \mu(\sigma)\left(a_{1}, \ldots, a_{n}\right)\right)$, and

- for each $c \in C_{s}, M_{c}=\mu(c)$.

For each $F M A L G(S, F, C)$-model $M$, we define an $\mathcal{I}(L)(S, F, C)$-model $(\mu, m)$, denoted $\beta_{(S, F, C)}^{\prime}(M)$, as follows:

- $\mu:(S, F, C) \rightarrow\left(S, F,\left(M_{s}\right)_{s \in S}\right)$ such that it consists of identities on $S$ and $F$, and $\mu(c)=M_{c}$ for each $c \in C_{s}$, and

- $m\left(a \prec \sigma\left(a_{1}, \ldots, a_{n}\right)\right)=M_{\sigma}\left(a_{1}, \ldots, a_{n}, a\right)$.

We note easily that for each $F M A L G(S, F, C)$-model $M, \beta_{(S, F, C)}\left(\beta_{(S, F, C)}^{\prime}(M)\right)=M$ and for each $\mathcal{I}(L)$ $(S, F, C)$-model $(\mu, m), \beta_{(S, F, C)}^{\prime}\left(\beta_{(S, F, C)}(\mu, m)\right)=(\mu, m)$.

Now we prove the equality (6) by recursion on the structure of the sentence $\rho$. Let us denote $\beta_{(S, F, C)}(\mu, m)$ by $M$. Let $\mu:(S, F, C) \rightarrow\left(S^{\prime}, F^{\prime}, C^{\prime}\right)$. We need the following lemma:

Lemma 4.1. For any extension $\chi:(S, F, C) \rightarrow(S, F, C+x)$ with $x$ variable, any $(S, F+C)$-term $t$, and any $\mu^{\prime}:(S, F, C+x) \rightarrow\left(S^{\prime}, F^{\prime}, C^{\prime}\right)$ such that $\mu=\chi ; \mu^{\prime}$ we have

$$
\left(\left(\mu^{\prime}, m\right) \models x \prec t\right)=M\left[t, \mu^{\prime}(x)\right] .
$$

Proof. We prove the lemma by recursion on the structure of the term $t$. Let $t=\sigma\left(t_{1}, \ldots, t_{n}\right)$ with $\sigma \in(F+C)_{w \rightarrow s}$.

When $\sigma \in F_{w \rightarrow s}$ : 


$$
\begin{aligned}
\left(\mu^{\prime}, m\right) \models x \prec \sigma\left(t_{1}, \ldots, t_{n}\right)= & \left.\left(\mu^{\prime}, m\right) \models(\exists \Upsilon)\left(x \prec \sigma\left(y_{1}, \ldots, y_{n}\right)\right) \wedge \bigwedge_{1 \leq i \leq n} y_{i} \prec t_{i}\right) \\
& (\text { definition of } x \prec t) \\
= & \bigvee\left\{\left(\mu^{\prime \prime}, m\right)\left(x \prec \sigma\left(y_{1}, \ldots, y_{n}\right) \wedge \bigwedge_{1 \leq i \leq n} y_{i} \prec t_{i}\right) \mid \mu^{\prime}=\Upsilon ; \mu^{\prime \prime}\right\} \\
& \left(\text { definition of }\left(\mu^{\prime}, m\right) \models-\right) \\
= & \bigvee\left\{m\left(\mu^{\prime}(x) \prec \mu(\sigma)\left(\mu^{\prime \prime}\left(y_{1}\right), \ldots, \mu^{\prime \prime}\left(y_{n}\right)\right)\right) \wedge \bigwedge_{1 \leq i \leq n}\left(\left(\mu^{\prime \prime}, m\right) \models y_{i} \prec t_{i}\right) \mid \mu^{\prime}=\Upsilon ; \mu^{\prime \prime}\right\} \\
& \left(\text { definition of }\left(\mu^{\prime}, m\right) \models-\right) \\
= & \bigvee\left\{M_{\sigma}\left(\mu^{\prime \prime}\left(y_{1}\right), \ldots, \mu^{\prime \prime}\left(y_{n}\right), \mu^{\prime}(x)\right) \wedge \bigwedge_{1 \leq i \leq n}\left(\left(\mu^{\prime \prime}, m\right) \models y_{i} \prec t_{i}\right) \mid \mu^{\prime}=\Upsilon ; \mu^{\prime \prime}\right\} \\
& \left(\text { definition of } M_{\sigma}\right) \\
= & \bigvee\left\{M_{\sigma}\left(\mu^{\prime \prime}\left(y_{1}\right), \ldots, \mu^{\prime \prime}\left(y_{n}\right), \mu^{\prime}(x)\right) \wedge \bigwedge_{1 \leq i \leq n} M\left[t_{i}, \mu^{\prime \prime}\left(y_{i}\right)\right] \mid \mu^{\prime}=\Upsilon ; \mu^{\prime \prime}\right\} \\
& (\operatorname{recursion} \text { hypothesis }) \\
= & M\left[\sigma\left(t_{1}, \ldots, t_{n}\right), \mu^{\prime}(x)\right]\left(\text { definition of } M\left[{ }_{-},\right]\right) .
\end{aligned}
$$

When $\sigma \in C_{s}$ :

$$
\begin{aligned}
& \left(\mu^{\prime}, m\right) \models x \prec \sigma=\left(\mu^{\prime}, m\right) \models(x=\sigma) \\
& =\left\{\begin{array}{l}
\top, \text { when } \mu^{\prime}(x)=\mu(\sigma), \\
\perp, \text { when } \mu^{\prime}(x) \neq \mu(\sigma)
\end{array} \quad \text { (definition of }\left(\mu^{\prime}, m\right) \text { and of } \text { CSen }_{0}\right. \text { ) } \\
& =\left\{\begin{array}{l}
\top, \text { when } M_{\sigma}=\mu^{\prime}(x), \\
\perp, \text { when } M_{\sigma} \neq \mu^{\prime}(x)
\end{array} \quad \text { (definition of } M_{\sigma}\right. \text { ) } \\
& \left.=M\left[\sigma, \mu^{\prime}(x)\right] \text { (definition of } M\left[{ }_{-},-\right]\right) \text {. }
\end{aligned}
$$

Now we get back to the proof of the proposition.

When $\rho$ is $t \prec t^{\prime}$ :

$$
\begin{aligned}
(\mu, m) \models t \prec t^{\prime} & =(\mu, m) \models(\forall \chi)\left(x \prec t \Rightarrow x \prec t^{\prime}\right) & & \text { (definition of } \alpha) \\
& =\bigwedge\left\{\left(\mu^{\prime}, m\right) \models x \prec t \Rightarrow x \prec t^{\prime} \mid \mu=\chi ; \mu^{\prime}\right\} & & \text { (definition of } \left.(\mu, m) \models_{-}\right) \\
& =\bigwedge\left\{\left(\left(\mu^{\prime}, m\right) \models x \prec t\right) \Rightarrow\left(\left(\mu^{\prime}, m\right) \models x \prec t^{\prime}\right) \mid \mu=\chi ; \mu^{\prime}\right\} & & \text { (definition of } \left.\left(\mu^{\prime}, m\right) \models_{-}\right) \\
& =\bigwedge\left\{M\left[t, \mu^{\prime}(x)\right] \Rightarrow M\left[t^{\prime}, \mu^{\prime}(x)\right] \mid \mu=\chi ; \mu^{\prime}\right\} & & \text { (Lemma 4.1) } \\
& =M \models t \prec t^{\prime} & & \text { (definition of } M[-,]) .
\end{aligned}
$$

When $\rho \in\{\top, \perp\}$ the proof is trivial. When $\rho$ is $\rho_{1} \star \rho_{2}$, with $\star \in\{\wedge, \vee, \Rightarrow\}$, and when $\rho$ is $(\forall X) \rho^{\prime}$ or $(\exists X) \rho^{\prime}$ the proofs are like in Prop. 4.1.

The following may be obtained as a straightforward instance of Prop. 2.1 via Prop. 4.3.

Corollary 4.2. For each FMALG signature $(S, F, C)$, each set $E$ of $F M A L G(S, F, C)$-sentences, each FMALG $(S, F, C)$-sentence e

$$
E \models{ }^{F M A L G} e \text { if and only if } \alpha_{(S, F, C)}(E) \models^{\mathcal{I}(L)} \alpha_{(S, F, C)}(e) .
$$

\section{Institution-theoretic properties of $\mathcal{I}(L)$}

In this section we show that the generic institution $\mathcal{I}(L)$ enjoys rather naturally a couple of properties that according to the tradition of institution-independent model theory (e.g. [10]) are of crucial importance for the development of an in-depth model theory at the abstract level of $\mathcal{I}(L)$. Given that $\mathcal{I}(L)$ may provide a generic model theory for various concrete mvl systems, the importance of these properties transfers to the level of these concrete mvl systems. 


\subsection{Model amalgamation}

The crucial role of model amalgamation for the semantics studies of formal specifications comes up in very many works in the area, a few early examples being [15, 27, 32, 34]; see also the recent reference [33]. The model amalgamation property is a necessary condition in very many institution-independent model theoretic results (see [10]), thus being one of the most desirable properties for an institution. It can be considered even as more fundamental than the satisfaction condition since in institutions with quantifications it is used in one of its weak forms in the proof of the satisfaction condition at the recursion step corresponding to quantifiers. (In this paper we have already met with this situation in the proof of Prop. 3.2.) At the concrete level of conventional model theory this property is implicit and because of this its importance is rather hidden. It is not to be confused with the much harder and less pervading amalgamation property that refers to the amalgamation of two elementary extensions of the same model, a property that appears within the context of a single signature.

Model amalgamation properties for institutions formalize the possibility of amalgamating models of different signatures when they are consistent on some kind of generalized 'intersection' of signatures. The following two definitions recalls corresponding concepts and terminology from the literature.

Definition 5.1 (Amalgamation square). A commutative square of signature morphisms

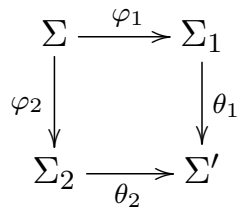

is an amalgamation square if and only if for each $\Sigma_{1}$-model $M_{1}$ and a $\Sigma_{2}$-model $M_{2}$ such that $\operatorname{Mod}\left(\varphi_{1}\right)\left(M_{1}\right)=$ $\operatorname{Mod}\left(\varphi_{2}\right)\left(M_{2}\right)$, there exists an unique $\Sigma^{\prime}$-model $M^{\prime}$, denoted $M_{1} \oplus_{\varphi_{1}, \varphi_{2}} M_{2}$, or $M_{1} \oplus M_{2}$ for short when there is no danger of ambiguity, such that $\operatorname{Mod}\left(\theta_{1}\right)\left(M^{\prime}\right)=M_{1}$ and $\operatorname{Mod}\left(\theta_{2}\right)\left(M^{\prime}\right)=M_{2}$.

In most of the institutions formalizing conventional or non-conventional logics, pushout squares of signature morphisms are model amalgamation squares [10].

Definition 5.2 (Semi-exactness). An institution has model amalgamation when each pushout square of signature morphisms is a model amalgamation square; it is semi-exact when this holds also for model homomorphisms.

The literature, especially the formal specification literature, considers also extensions of model amalgamation from pushouts to arbitrary co-limits, however amalgamation for pushout squares is by far the most important case.

Proposition 5.1. The institution $\mathcal{I}(L)$ is semi-exact.

Proof. We consider a pushout square of signature morphisms like below and a $\Sigma_{1}$-model $\left(\mu_{1}, m_{1}\right)$ and a $\Sigma_{2}$-model $\left(\mu_{2}, m_{2}\right)$ such that $\varphi_{1} ; \mu_{1}=\varphi_{2} ; \mu_{2}$.

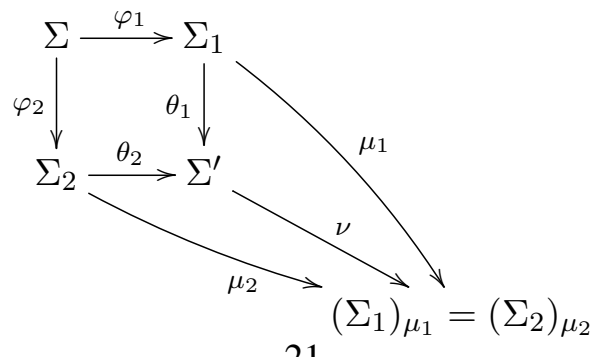


This implies $\left(\Sigma_{1}\right)_{\mu_{1}}=\left(\Sigma_{2}\right)_{\mu_{2}}$ and $m_{1}=m_{2}$. By the pushout property there exists an unique $\nu: \Sigma^{\prime} \rightarrow$ $\left(\Sigma_{i}\right)_{\mu_{i}}$ such that $\theta_{i} ; \nu=\mu_{i}$ for $i \in\{1,2\}$. Then $\left(\nu, m_{i}\right)$ is the unique amalgamation of $\left(\mu_{1}, m_{1}\right)$ and $\left(\mu_{2}, m_{2}\right)$. We have thus shown the model amalgamation property at the level of the models.

Now let us consider homomorphisms $h_{k}:\left(\mu_{k}, m_{k}\right) \rightarrow\left(\mu_{k}^{\prime}, m_{k}^{\prime}\right), k \in\{1,2\}$, such that $\operatorname{Mod}\left(\varphi_{1}\right)\left(h_{1}\right)=$ $\operatorname{Mod}\left(\varphi_{2}\right)\left(h_{2}\right)$. This means $\left(h_{1}=h_{2}\right):\left(\left(\Sigma_{1}\right)_{\mu_{1}}=\left(\Sigma_{2}\right)_{\mu_{2}}\right) \rightarrow\left(\left(\Sigma_{1}\right)_{\mu_{1}^{\prime}}=\left(\Sigma_{2}\right)_{\mu_{2}^{\prime}}\right)$ and also $m_{1}=$ $m_{2}$ and $m_{1}^{\prime}=m_{2}^{\prime}$. Let $(\nu, m)=\left(\mu_{1}, m_{1}\right) \oplus\left(\mu_{2}, m_{2}\right)$ and $\left(\nu^{\prime}, m^{\prime}\right)=\left(\mu_{1}, m_{1}\right) \oplus\left(\mu_{2}, m_{2}\right)$. Then $\left(h_{1}=h_{2}\right):\left(\left(\Sigma_{1}\right)_{\mu_{1}}=\left(\Sigma_{2}\right)_{\mu_{2}}=\Sigma_{\nu}^{\prime}\right) \rightarrow\left(\left(\Sigma_{1}\right)_{\mu_{1}^{\prime}}=\left(\Sigma_{2}\right)_{\mu_{2}^{\prime}}=\Sigma_{\nu^{\prime}}^{\prime}\right)$ is the unique homomorphism $h:(\nu, m) \rightarrow\left(\nu^{\prime}, m^{\prime}\right)$ such that $\operatorname{Mod}\left(\theta_{k}\right)(h)=h_{k}$ for $k \in\{1,2\}$.

\subsection{The method of diagrams}

In conventional model theory the method of diagrams is one of the most important methods. The institution-independent method of diagrams pervades the development of a lot of model theoretic results at the level of abstract institutions, many of these being presented in [10]. In the form presented here it has been introduced at the level of institution-independent model theory in [9] as a categorical property which formalizes the idea that the class of model homomorphisms from a model $M$ can be represented (by a natural isomorphism) as a class of models of a theory in a signature extending the original signature with syntactic entities determined by $M$. This can be seen as a coherence property between the semantic and the syntactic structures of the institution. By following the basic principle that a structure is rather defined by its homomorphisms (arrows) than by its objects, the semantic structure of an institution is given by its model homomorphisms. On the other hand the syntactic structure of an institution is based upon its corresponding concept of atomic sentence.

Below we recall the main definition from $[9,10]$.

Definition 5.3 (The method of diagrams). An institution $\mathcal{I}$ has diagrams when for each signature $\Sigma$ and each $\Sigma$-model $M$, there exists a signature $\Sigma_{M}$ and a signature morphism $\iota_{\Sigma}(M): \Sigma \rightarrow \Sigma_{M}$, functorial in $\Sigma$ and $M$, and a set $E_{M}$ of $\Sigma_{M}$-sentences such that $\operatorname{Mod}\left(\Sigma_{M}, E_{M}\right)$ and the comma category $M / \operatorname{Mod}(\Sigma)$ are naturally isomorphic, i.e. the following diagram commutes by the isomorphism $i_{\Sigma, M}$ that is natural in $\Sigma$ and $M$

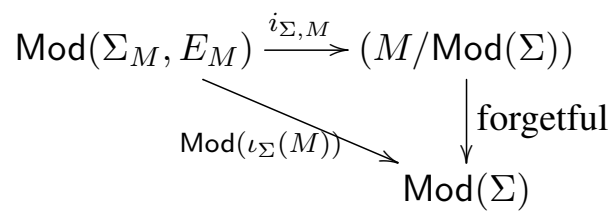

The signature morphism $\iota_{\Sigma}(M): \Sigma \rightarrow \Sigma_{M}$ is called the elementary extension of $\Sigma$ via $M$ and the set $E_{M}$ of $\Sigma_{M}$-sentences is called the diagram of the model $M$.

The functoriality of $\iota$ means that for each signature morphism $\varphi: \Sigma \rightarrow \Omega$ and each $\Sigma$-model homomorphism $h: M \rightarrow \operatorname{Mod}(\varphi)(N)$, there exists a morphism $\iota_{\varphi}(h): \Sigma_{M} \rightarrow \Omega_{N}$ such that

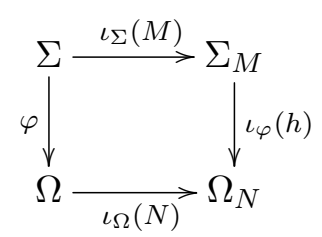

commutes, $\iota_{\varphi}(h) ; \iota_{\varphi^{\prime}}\left(h^{\prime}\right)=\iota_{\varphi ; \varphi^{\prime}}\left(h ; \operatorname{Mod}(\varphi)\left(h^{\prime}\right)\right)$ (for another $\varphi^{\prime}: \Omega \rightarrow \Theta$ and $\Omega$-model homomorphism $\left.h^{\prime}: N \rightarrow \operatorname{Mod}\left(\varphi^{\prime}\right)(P)\right)$, and such that $\iota_{1_{\Sigma}}\left(1_{M}\right)=1_{\Sigma_{M}}$. Moreover we require that $E_{N} \models$ $\operatorname{Sen}\left(\iota_{\varphi}(h)\right)\left(E_{M}\right)$. 
The naturality of $i$ means that for each signature morphism $\varphi: \Sigma \rightarrow \Omega$ and each $\Sigma$-model homomorphism $h: M \rightarrow \operatorname{Mod}(\varphi)(N)$ the following diagram commutes:

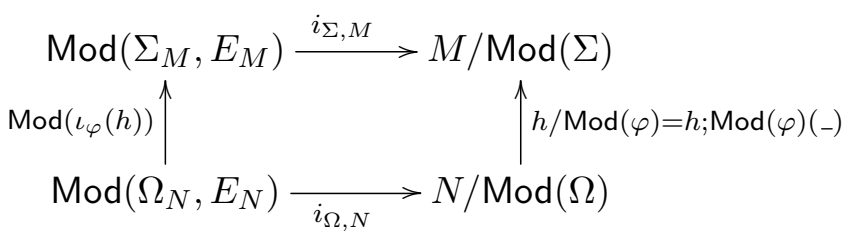

The following result shows that $\mathcal{I}(L)$ is equipped rather naturally with a system of diagrams.

Proposition 5.2. $\mathcal{I}(L)$ has diagrams.

Proof. For any $\mathcal{I}(L) \Sigma$-model $(\mu, m)$, its diagram $\left(\Sigma_{(\mu, m)}, E_{(\mu, m)}\right)$ is defined by

$$
\begin{aligned}
-\Sigma_{(\mu, m)} & =\Sigma_{\mu}, \text { and } \\
-E_{(\mu, m)} & =\left\{(\rho, m(\rho)) \mid \rho \in \mathrm{FSen}\left(\Sigma_{\mu}\right)\right\} .
\end{aligned}
$$

For each signature morphism $\varphi: \Sigma \rightarrow \Omega$ and each model homomorphism $h:(\mu, m) \rightarrow(\varphi ; \nu, n)$
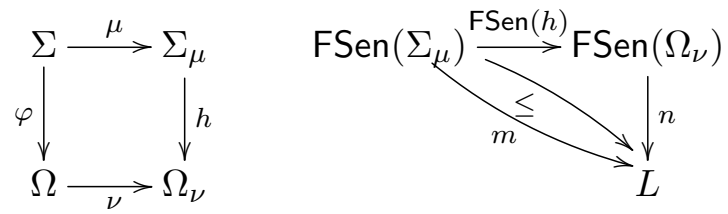

we define $\iota_{\varphi}(h)=h$.

That $E_{(\nu, n)} \models h\left(E_{(\mu, m)}\right)$ follows from the fact that for each $\rho \in \mathrm{FSen}\left(\Sigma_{\mu}\right)$ we have that $(h(\rho), n(h(\rho))) \models$ $(h(\rho), m(\rho))$ since $m \leq \mathrm{FSen}(h) ; n$ by the homomorphism condition for $h:(\mu, m) \rightarrow(\nu, n)$.

For each $\mathcal{I}(L) \Sigma$-model $(\mu, m)$ we define

$$
i_{\Sigma,(\mu, m)}\left(\mu^{\prime}, m^{\prime}\right)=\left(\mu^{\prime}:(\mu, m) \rightarrow\left(\mu ; \mu^{\prime}, m^{\prime}\right)\right) .
$$

The following calculation shows that $\left(\mu^{\prime}, m^{\prime}\right) \models E_{(\mu, m)}$ if and only if $\mu^{\prime}$ is a homomorphism $(\mu, m) \rightarrow$ $\left(\mu ; \mu^{\prime}, m^{\prime}\right)$.

$$
\begin{aligned}
& \left(\mu^{\prime}, m^{\prime}\right) \models E_{(\mu, m)} \text { if and only if } \quad\left(\mu^{\prime}, m^{\prime}\right) \models(\rho, m(\rho)) \text { for all } \rho \in \mathrm{FSen}\left(\Sigma_{\mu}\right) \\
& \text { (definition of } \left.E_{(\mu, m)}\right) \\
& \text { if and only if } m(\rho) \leq\left(\left(\mu^{\prime}, m^{\prime}\right) \models \rho\right) \text { for all } \rho \in \mathrm{FSen}\left(\Sigma_{\mu}\right) \\
& \text { (definition of } \models \text { ) } \\
& \text { if and only if } m(\rho) \leq m^{\prime}\left(\operatorname{FSen}\left(\mu^{\prime}\right)(\rho)\right) \text { for all } \rho \in \mathrm{FSen}\left(\Sigma_{\mu}\right) \\
& \text { (definition of }\left(\mu^{\prime}, m^{\prime}\right) \models{ }_{-} \text {) } \\
& \text { if and only if } \quad \mu^{\prime} \text { is homomorphism }(\mu, m) \rightarrow\left(\mu^{\prime}, m^{\prime}\right) \\
& \text { (definition of homomorphism). }
\end{aligned}
$$

Note that the inverse $i_{\Sigma,(\mu, m)}^{-1}$ is given by $i_{\Sigma,(\mu, m)}^{-1}\left(\mu^{\prime}\right)=\left(\mu^{\prime}, m^{\prime}\right)$.

The naturality of $i$ is proved by the calculations below. Let $\left(\nu^{\prime}, n^{\prime}\right)$ be any $\mathcal{I}(L) \Omega_{(\nu, n)}$-model such that $\left(\nu^{\prime}, n^{\prime}\right) \models E_{(\nu, n)}$. 


$$
\begin{aligned}
\left(h / \operatorname{Mod}^{\mathcal{I}(L)}(\varphi)\right)\left(i_{\Omega,(\nu, n)}\left(\nu^{\prime}, n^{\prime}\right)\right)= & \left(h / \operatorname{Mod}^{\mathcal{I}(L)}(\varphi)\right)\left(\nu^{\prime}:(\nu, n) \rightarrow\left(\nu ; \nu^{\prime}, n^{\prime}\right)\right) \\
& \left(\text { definition of } i_{\Omega,(\nu, n)}\right) \\
= & (h /-)\left(\nu^{\prime}:(\varphi ; \nu, n) \rightarrow\left(\varphi ; \nu ; \nu^{\prime}, n^{\prime}\right)\right) \\
& (\text { definition of model reducts in } \mathcal{I}(L)) \\
= & \left.h ; \nu^{\prime}:(\mu ; h, m) \rightarrow\left(\mu ; h ; \nu^{\prime}, n^{\prime}\right)\right) \\
& (\text { definition of } h /- \text { and } \varphi ; \nu=\mu ; h) \\
= & i_{\Sigma,(\mu, m)}\left(h ; \nu^{\prime}, n^{\prime}\right)\left(\text { definition of } i_{\Sigma,(\mu, m)}\right) \\
= & i_{\Sigma,(\mu, m)}\left(\operatorname{Mod}^{\mathcal{I}(L)}\left(\iota_{\varphi}(h)\left(\nu^{\prime}, n^{\prime}\right)\right)\right) \\
& \left(\text { definition of model reducts in } \mathcal{I}(L) \text { and of } \iota_{\varphi}(h)\right) .
\end{aligned}
$$

\section{Conclusions and Future Research}

In this paper we have introduced a generic institution for mvl, namely $\mathcal{I}(L)$, that includes an mvl specific abstract model theory, a syntax built generically over a fully abstract atomic syntax, and a satisfaction relation defined in the style of Tarski. $\mathcal{I}(L)$ may serve as a general framework for defining uniformly mvl systems over various syntaxes. We have illustrated this with traditional first order mvl, with its propositional and second order variations, and with fuzzy multi-algebras. Moreover our work allows to develop an in-depth mvl specific model theory that is abstract and syntax independent and which therefore may be uniformly applicable by instantiation to various concrete mvl systems. We have taken a first important step in this direction by showing that our generic mvl institution enjoys a couple of properties of crucial importance in institution-independent model theory, namely model amalgamation and the method of diagrams.

We plan to develop further model theory results and methods at the generic abstract level of $\mathcal{I}(L)$, including the method of ultraproducts, studies of quasi-varieties and of existence of initial semantics. The latter is expected to lift results that have been developed at the concrete level of traditional mvl [12] to the abstract level of $\mathcal{I}(L)$.

Another interesting further research direction is the definition of already known or new concrete mvl systems as instances of $\mathcal{I}(L)$, possibly involving the more sophisticated embeddings based upon the socalled 'theoroidal comorphisms' of [21, 29] that map signatures to theories rather than plain signatures (as done in our paper). An illustration of such use of theoroidal embeddings would be the embedding of classical (two-valued) partial algebras into $\mathcal{I}(L)$. This is similar to our embedding of $F M A L G$ into $\mathcal{I}(L)$, however in the case of the partial algebras one would like to make sure that the result of applying an operation to its arguments gives at most one result. This requires for each operation symbol $\sigma$ a Horn sentence of the form $\left(\forall \bar{x}, y_{1}, y_{2}\right)\left(\sigma(\bar{x})=y_{1}\right) \wedge\left(\sigma(\bar{x})=y_{2}\right) \Rightarrow\left(y_{1}=y_{2}\right)$.

\section{Acknowledgements}

This work has been supported by a grant of the Romanian National Authority for Scientific Research, CNCS-UEFISCDI, project number PN-II-ID-PCE-2011-3-0439.

\section{References}

[1] Edigio Astesiano, Michel Bidoit, Hélène Kirchner, Berndt Krieg-Brückner, Peter Mosses, Don Sannella, and Andrzej Tarlecki. CASL: The common algebraic specification language. Theoretical Computer Science, 286(2):153-196, 2002.

[2] Jean-Yves Béziau. 13 questions about universal logic. Bulletin of the Section of Logic, 35(2/3):133-150, 2006.

[3] Jean-Yves Béziau, editor. Universal Logic: an Anthology. Studies in Universal Logic. Springer Basel, 2012.

[4] Peter Burmeister. Partial algebra - an introductory survey. Algebra Universalis, 15:306-358, 1982. 
[5] Rod Burstall and Joseph Goguen. The semantics of Clear, a specification language. In Dines Bjorner, editor, 1979 Copenhagen Winter School on Abstract Software Specification, volume 86 of Lecture Notes in Computer Science, pages 292-332. Springer, 1980.

[6] Manuel Clavel, Francisco Durán, Steven Eker, Patrick Lincoln, Narciso Martí-Oliet, José Meseguer, and Carolyn Talcott. All About Maude - A High-Performance Logical Framework, volume 4350 of Lecture Notes in Computer Science. Springer, 2007.

[7] Denisa Diaconescu. Model theory for Multiple-valued Logics. Master’s thesis, Şcoala Normală Superioară Bucureşti, 2009.

[8] Răzvan Diaconescu. Grothendieck institutions. Applied Categorical Structures, 10(4):383-402, 2002. Preliminary version appeared as IMAR Preprint 2-2000, ISSN 250-3638, February 2000.

[9] Răzvan Diaconescu. Elementary diagrams in institutions. Journal of Logic and Computation, 14(5):651-674, 2004.

[10] Răzvan Diaconescu. Institution-independent Model Theory. Birkhäuser, 2008.

[11] Răzvan Diaconescu. Quasi-boolean encodings and conditionals in algebraic specification. Journal of Logic and Algebraic Programming, 79(2):174-188, 2010.

[12] Răzvan Diaconescu. On quasi-varieties of multiple valued logic models. Mathematical Logic Quarterly, 57(2):194-203, 2011.

[13] Răzvan Diaconescu. Structural induction in institutions. Information and Computation, 209(9):1197-1222, 2011.

[14] Răzvan Diaconescu and Kokichi Futatsugi. CafeOBJ Report: The Language, Proof Techniques, and Methodologies for Object-Oriented Algebraic Specification, volume 6 of AMAST Series in Computing. World Scientific, 1998.

[15] Răzvan Diaconescu, Joseph Goguen, and Petros Stefaneas. Logical support for modularisation. In Gerard Huet and Gordon Plotkin, editors, Logical Environments, pages 83-130. Cambridge, 1993. Proceedings of a Workshop held in Edinburgh, Scotland, May 1991.

[16] Patrick Eklund and Robert Helgesson. Monadic extensions of institutions. Fuzzy Sets and Systems, 161:2354-2368, 2010.

[17] Nikolaos Galatos, Peter Jipsen, Tomasz Kowalski, and Hiroakira Ono. Residuated Lattices: An Algebraic Glimpse at Substructural Logics. Elsevier, 2007.

[18] Kurt Gödel. Zum intuitionistischen aussagenkalkül. Anzeiger Akademie der Wissenschaften Wien, Mathnaturwiss. Klasse 69:65-66, 1932.

[19] Joseph Goguen and Rod Burstall. Institutions: Abstract model theory for specification and programming. Journal of the Association for Computing Machinery, 39(1):95-146, 1992.

[20] Joseph Goguen and José Meseguer. Models and equality for logical programming. In Hartmut Ehrig, Giorgio Levi, Robert Kowalski, and Ugo Montanari, editors, Proceedings, TAPSOFT 1987, volume 250 of Lecture Notes in Computer Science, pages 1-22. Springer, 1987.

[21] Joseph Goguen and Grigore Roşu. Institution morphisms. Formal Aspects of Computing, 13:274-307, 2002.

[22] Petr Hájek. Metamathematics of Fuzzy Logic. Kluwer, 1998.

[23] Heinrich Hussmann. Nondeterminism in Algebraic Specifications and Algebraic Program. Birkhaüser, 1993.

[24] Yngve Lamo. The Institution of Multialgebras - a general framework for algebraic software development. PhD thesis, University of Bergen, 2003.

[25] Saunders Mac Lane. Categories for the Working Mathematician. Springer, second edition, 1998.

[26] J. Łukasiewicz. Philosophische bemerkungen zu mehrwertigen systemen des aussagenkalküls. Comptes Rendus Séances Société des Sciences et Lettres Varsovie, (cl. III(23):51-77, 1930.

[27] José Meseguer. General logics. In H.-D. Ebbinghaus et al., editors, Proceedings, Logic Colloquium, 1987, pages $275-329$. North-Holland, 1989.

[28] José Meseguer. Membership algebra as a logical framework for equational specification. In F. Parisi-Pressice, editor, Proc. WADT'97, volume 1376 of Lecture Notes in Computer Science, pages 18-61. Springer, 1998.

[29] Till Mossakowski. Different types of arrow between logical frameworks. In F. Meyer auf der Heide and B. Monien, editors, Proc. ICALP 96, volume 1099 of Lecture Notes in Computer Science, pages 158-169. Springer Verlag, 1996.

[30] E.L. Post. Introduction to a general theory of elementary propositions. Amer. J. Math., 43:163-185, 1921.

[31] Florian Rabe. A framework for combining model and proof theory. Mathematical Structures in Computer Science, 2012. Submitted.

[32] Donald Sannella and Andrzej Tarlecki. Specifications in an arbitrary institution. Information and Control, 76:165-210, 1988.

[33] Donald Sannella and Andrzej Tarlecki. Foundations of Algebraic Specifications and Formal Software Development. Springer, 2012.

[34] Andrzej Tarlecki. On the existence of free models in abstract algebraic institutions. Theoretical Computer Science, 37:269304, 1986.

[35] Michał Walicki. Algebraic Specification of Nondeterminism. PhD thesis, Department of Informatics, University of Bergen, 1993.

[36] Michał Walicki and Sigurd Meldal. Algebraic approaches to nondeterminism - an overview. ACM Computing Surveys, 29, 
1997.

[37] Morgan Ward and Robert Dilworth. Residuated lattices. Trans. Amer. Math. Soc., 45:335-354, 1939. 